\title{
We are not alone: Extraterrestrial Technological Life in our Galaxy
}

Strom, Robert G

Department of Planetary Sciences, Lunar and Planetary Laboratory, University of Arizona Tucson, Arizona, USA

*Corresponding author: Strom RG, Department of Planetary Sciences, Lunar and Planetary Laboratory, University of Arizona Tucson, Arizona, USA, Tel: +39348 2650891; E-mail: rstrom@lpl.arizona.edu

Rec date: September 27, 2015; Acc date: October 31, 2015; Pub date: November 3, 2015

Copyright: (C) 2015 Strom RG. This is an open-access article distributed under the terms of the Creative Commons Attribution License, which permits unrestricted use, distribution, and reproduction in any medium, provided the original author and source are credited.

\begin{abstract}
This paper is an evaluation of the number of technological civilizations presently in our Milky Way galaxy. The current estimate of the present number of Earth-like planets $( \pm 25 \%$ of Earth's radius) in the habitable region of Sunlike stars is about 6.6 billion. Using a modified Drake Equation, "optimistic", "pessimistic" and "unlikely" estimates of six constraints indicate that about 40,000 technological civilizations are presently in our galaxy for a "pessimistic evaluation". Even "unlikely" values that are half the "pessimistic" values give over 600 technological civilizations in the galaxy. It would take a completely unrealistic value of $2.5 \%$ for each constraint to get just 1 technology (us). Therefore, we are almost surely not alone in our Milky Way galaxy. Furthermore, almost all of the extra-solar system technologies are probably much further advanced than ours, i.e., they probably began thousands to millions of years before our Industrial Revolution. It is possible, but highly uncertain, that we have been visited by one or more of these civilizations.
\end{abstract}

Keywords: Milky Way galaxy; Terrestrial planets; Extraterrestrial life; Intelligent life; Solar System; Technological life

\section{Introduction}

The recent finding [1] that our Milky Way galaxy contains about 8.8 billion Earth-like planets orbiting Sun-like stars in their habitable zones has surprised and perhaps shocked the science community. Discoveries of exoplanets in our galaxy continue to increase on almost a daily basis. To date (2014) over 4000 planets have been discovered, and for the first time a planet almost the same size as the Earth has been discovered orbiting a red dwarf in its habitable zone. These findings have raised the question of how many technological civilizations there are in our galaxy, much less in the universe and beyond. This paper discusses the possible current number of technological civilizations in our Milky Way galaxy and our chances of communicating with them.

\section{Part 1: The Milky Way galaxy}

The Milky Way galaxy is the home of our Solar System. It is a barred spiral galaxy about 100,000-120,000 light-years in diameter containing about $300 \pm 100$ billion stars. It may contain at least as many planets. Its thickness is about 1,000 light-years. The age of our galaxy is about 13.2 billion years. Figure 1a is a mosaic of Earth-based images showing the plane of the Milky Way galaxy.

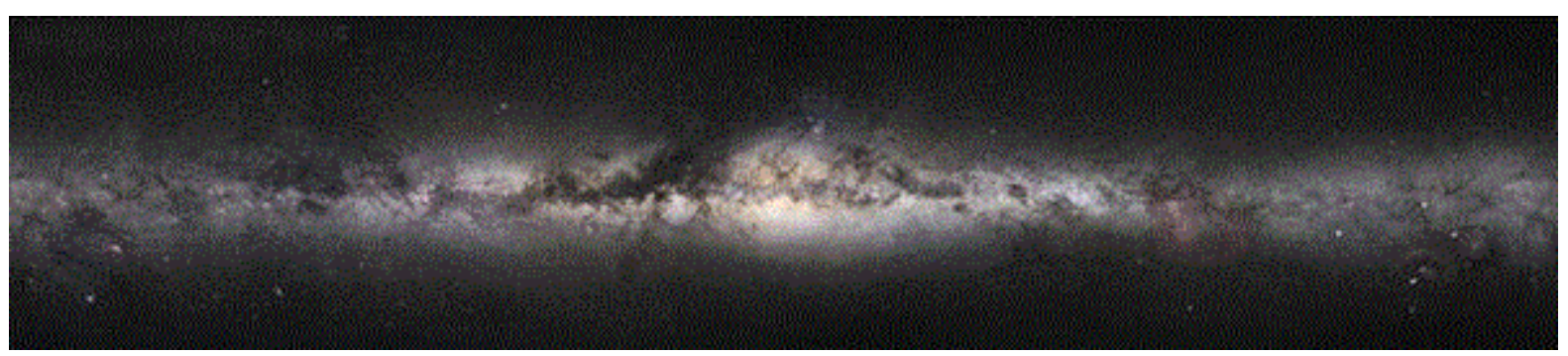

Figure 1(a): Image mosaic of the plane of our Milky Way galaxy.

Solar systems are thought to form from protoplanetary discs of gas and dust around newly formed stars. They form from the coalescence of clumps of material that sweep out lanes forming dark rings in the disc. Figure $1 \mathrm{~b}$ is an image of a protoplanetary disc probably during planet formation.

The present-day formation rate of stars with the Sun's mass is estimated to be $0.68-1.45 \mathrm{Msun} / \mathrm{yr}$ [2]. If the present rate is similar to the past rate, then about 9-19 billion solar mass stars formed between 13.2 billion years ago and the present. The lifetimes of stars depends on their mass (Figure 1c). Stars with similar masses to our Sun have lifetimes of about 10 billion years. Therefore, between 2.2 and 4.6 billion of those stars have exceeded their lifetimes and no longer exist. A technological civilization on any planets around these stars would have been destroyed, moved to some other habitable planet around another star or continuously live in an artificial spacecraft.

Recently a very old star with Earth-sized planets has been discovered by the Kepler spacecraft [3]. Kepler-444, a metal-poor Sunlike star has an age of 11.2 $\pm 1.0 \mathrm{Gyr}$ and 5 planets with sizes between 
Citation: Strom, Robert G (2015) We are not alone: Extraterrestrial Technological Life in our Galaxy. Astrobiol Outreach 3: 144. doi:

those of Mercury and Venus. Therefore, Kepler-444 formed when the Universe was less than $20 \%$ of its current age. This indicates there were enough heavy elements in the early history of the Universe to form terrestrial-like planets. Therefore, Earth-like planets formed throughout most the Universe 13.8 Gyr history. Figure 1d is a diagram showing a comparison of the Kepler-444 system to other planetary systems.

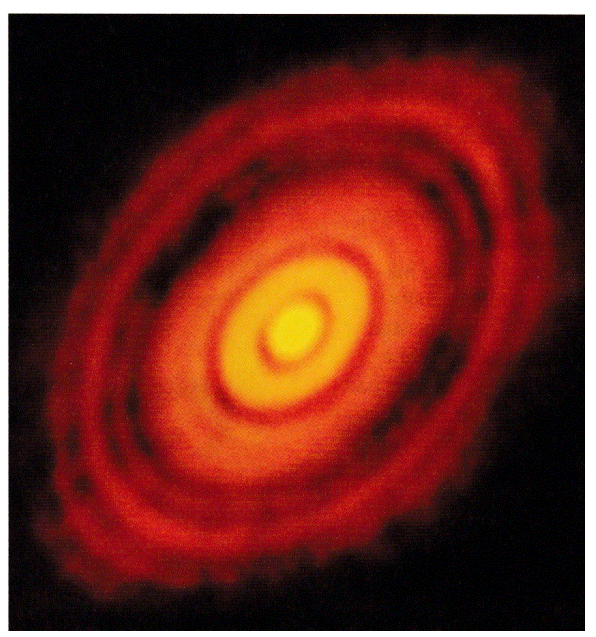

Figure 1(b): This image of a protoplanetary disc is the Sun-like star HI.Tan that is $450 \mathrm{LY}$ from Earth. It was taken by the Atacama Large Millimeter/submillimeter Array in Chili on Nov 6, 2014.

Lifetime of Stars Relative to Mass of Sun

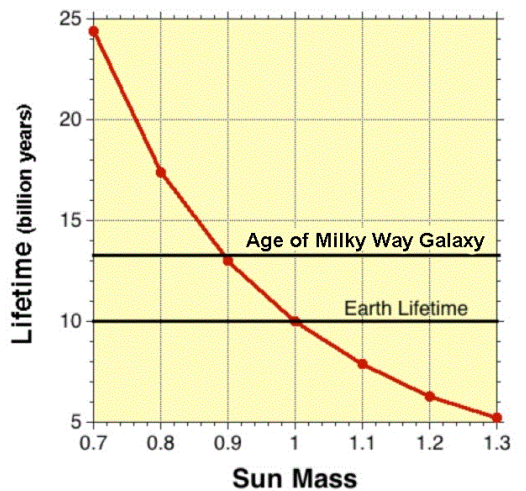

Figure 1(c): This is a plot of stellar lifetimes as a function of solar masses. The Sun and Earth's lifetime is 10 billion years for one solar mass.

The Solar System is located in the inner edge of a spiral-shaped concentration of gas and dust called the Orion-Cygnus Arm (Figure 1e and 1f). It is about 28,000 light-years from the galactic center; located about 75,000 light years from the farthest edge and about 25,000 light years from the nearest edge (Figure 1e). The light we see today from the galactic center and nearest edge was emitted long before civilization began about 12,000 years ago.

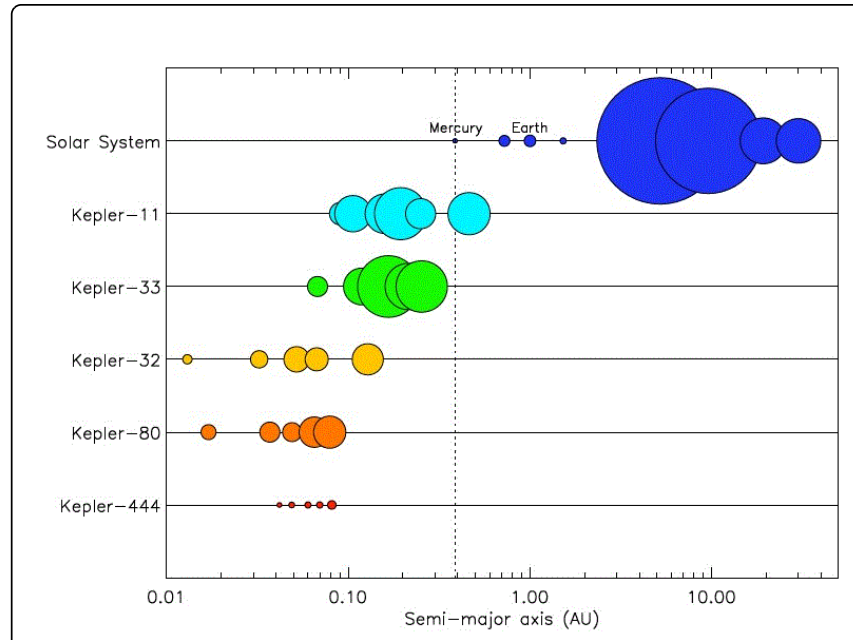

Figure 1(d): Semi-major axes of planets belonging to the highly compact multiple-planet systems of other Kepler systems and our solar system. The vertical dotted line marks the semi- major axis of Mercury. Symbol size is proportional to planet diameter [3].

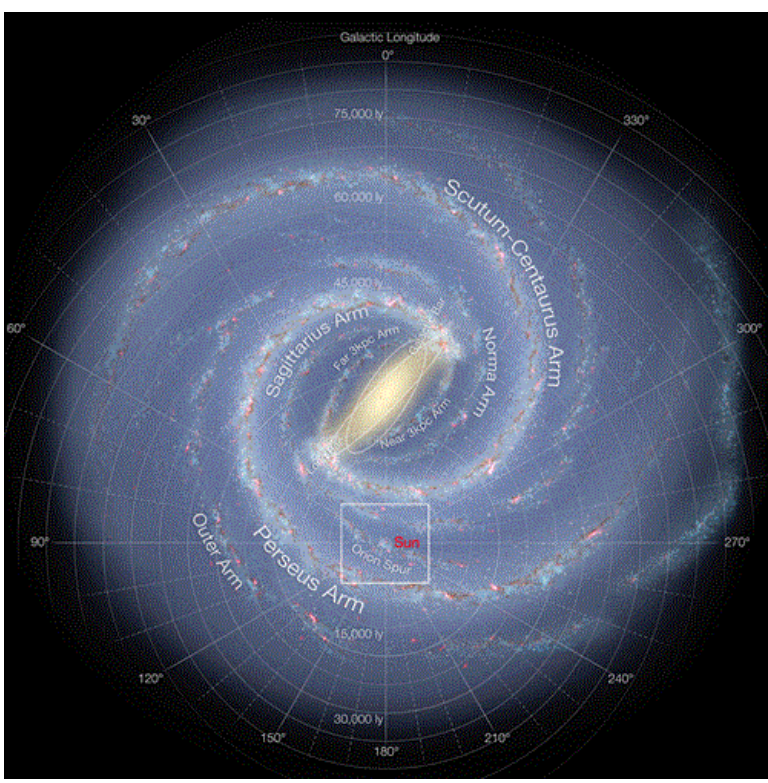

Figure 1(e): Artist's rendition of the Milky Way galaxy. Our Solar System is shown by the red label "Sun" which is $\sim 28,000$ light-years from the galactic center. The distance scale is centered on the Sun with galactic longitudes at $30^{\circ}$ intervals. The white square is the location of Figure 1f.

Figure if is an enlargement of the galactic region at the Sun's location shown in Figure 1e. The diameter of the smaller circle centered on the Sun is about $1600 \mathrm{LY}$ and the diameter of the large circle is 10,000 LY. Our first radio transmission on a large scale began 92 years ago. That signal has traveled only a diameter of $\sim 184$ LY or about $12 \%$ of the small circle diameter shown in Figure 1f. Therefore, any technological society greater than a radius of about $92 \mathrm{LY}$ from Earth probably does not yet know we exist; that would include over 
99.9\% of the galaxy. Furthermore, the radio emissions would become weaker as they travelled to great distance until they would be undetectable from the background radiation. The size of the white dot at the center of the small circle is the present distance that our radio waves have traveled in the last 92 years.

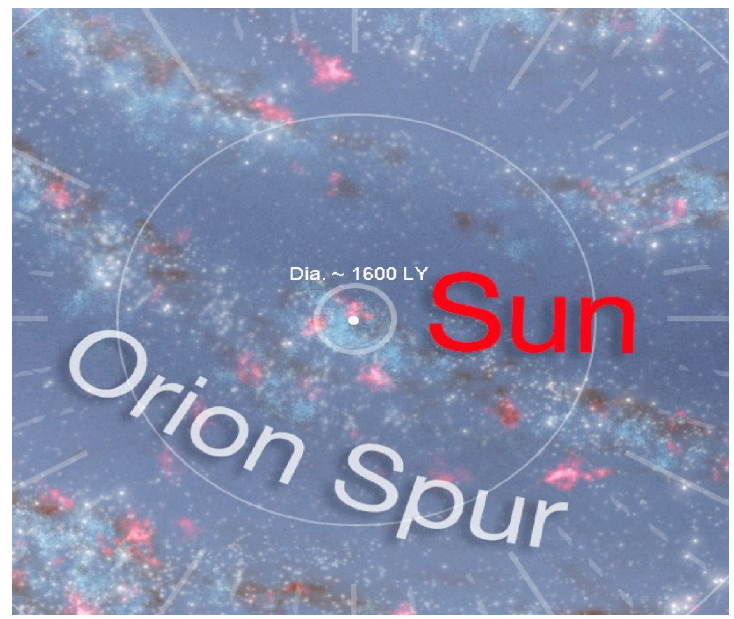

Figure 1(f): This enlargement of Figure 1e shows in more detail the region in the vicinity of the Sun. The diameter of the small circle is about $1600 \mathrm{LY}$ and the large circle is $10,000 \mathrm{LY}$ in diameter. The white dot in the center is the distance our radio waves have traveled in the 92 years since we began major transmissions.

It is unlikely that another technological civilization would be able to detect our radio transmissions unless they are relatively close to our Sun and have very sensitive equipment. The most likely means of detecting us is by our light emission (Part 6). If we sent 1015watt laser pulses aimed at a given star with an Earth-like planet in the habitable zone, then they may be able to detect us, but it would take a very long time to send them long distances and an equally long time if they replied. We could be communicatively isolated in our own galaxy unless the civilizations' star is relatively close (Part 6).

\section{Part 2: What is a Habitable Zone?}

A habitable zone is a region of space around a star where conditions are favorable for life, e.g., an environment favorable for surface water. Planets and moons in these regions are the likeliest candidates to be habitable. Our Sun has a temperature of about 5800 K. For stars cooler than our Sun (M dwarfs, also known as red dwarfs, at 3000-4000 K) the region is closer in. For hotter stars (A dwarfs at 10,000 K) the region is much farther away from the star (Figure 2a).

The habitable zone surrounding our Sun is the region where surface water is stable and the environment is favorable for the formation of life. It is the zone where it is not too hot or too cold for life to exist; sometimes called the "Goldie Locks Zone". However, it should be understood that just because an Earth-like planet occurs in the habitable zone does not mean water on its surface will last long enough for advanced life to form. Mars and Venus are examples as discussed in Part 3. For our Sun the habitable zone is thought to be between the orbits of Venus and Mars, or 0.72 to 1.5 Astronomical Units (1 AU is the distance from the Earth to the Sun).

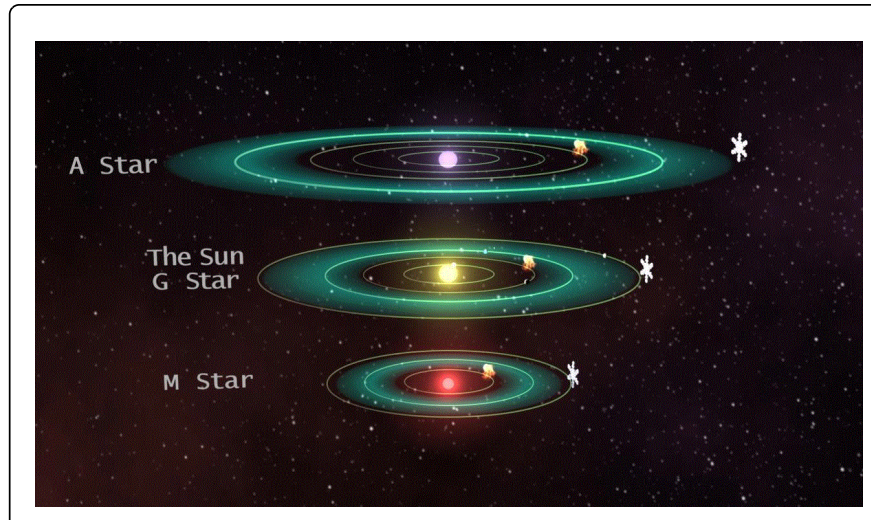

Figure 2(a): This diagram shows the habitable regions around A, G (Sun) and $M$ stars. The fuzzy green regions around the stars are their habitable zones. For our Sun the inner symbol represents the orbit of Venus, the prominent green ellipse is the Earth, and the outer yellow ellipse is the orbit of Mars. The A star shows the equivalent orbits of Venus and Earth, and the $M$ star shows the equivalent orbits of Venus, Earth and Mars. The habitable zone may extend from the orbit of Venus to that of Mars as discussed below (Courtesy of NASA).

A recent study [4] suggests that the habitable zone in our Solar System is smaller than previous estimates. It may only begin about 0.9 $\mathrm{AU}$ instead of $0.72 \mathrm{AU}$. This estimate is partly based on the observation of Venus being an extremely hot, desiccated planet. If this smaller estimate of the habitable zone is correct then it would lower the number of estimated Earth-like planets in the habitable zone by about a factor of 2. Therefore, instead of 8.8 billion Earth-like planets in the habitable zone of Sun-like stars, there may be only about 4.4 billion. For planets similar in size to Earth there would be about 3.3 billion. However, additional calculations are required to determine whether the results of this study are robust. Also, this new estimate of the habitable zone does not take into account the geologic evolution and consequent atmospheric evolution of an Earth-like planet. For example, Venus' extreme heat and desiccation are not due to its position relative to the Sun, but to its geological evolution, as discussed in Part 3. Without that geologic history, but one more like the Earth's, Venus today might be very similar to present Earth.

\section{Estimated earth-like planets in the habitable zone}

A study by Petigura, et al. [1] using the Kepler mission data has estimated that in our galaxy about 1 in 5 stars, or about 40 billion stars, are like our Sun in size, color, and age. It is possible that there could be as many as 50 billion of these stars.

The Kepler telescope continuously monitored the brightness of about 150,000 nearby stars over long periods to detect slight periodic dimming that could indicate the repeated transit of a planet across the face of its host star. The stars in a northern part of the sky were monitored every half hour for a period of four years. The search found 603 planets transiting sun-like stars. This is a minimum because planets in orbits out of the line of sight would not be detected because they do not transit across a star's face. Figure $2 b$ shows the detected planets plotted against planet radius and orbital period (a) or stellar irradiation flux (b). Figure $2 \mathrm{c}$ shows the estimated fraction of Sun-like stars having planets of different sizes (left) and orbital period (right). 
Citation: Strom, Robert G (2015) We are not alone: Extraterrestrial Technological Life in our Galaxy. Astrobiol Outreach 3: 144. doi:

Page 4 of 18

From these data the authors of the study estimate about $22 \pm 8 \%$ of the 40 billion Sun-like stars in our Milky Way galaxy have Earth-like planets (silicate mantles and crusts with iron cores) in the habitable zone. This amounts to about $8.8 \pm 3.2$ billion Earth-like planets in a zone where liquid surface water is stable. The closest Earth-like planet is estimated to be about $12 \mathrm{LY}$ away or about $1.13 \times 10^{14}$ kilometers.
The planets are estimated to be between 1 and 16 Earth radii with most between 1 and 4 Earth radii (Figure 2c). It is estimated that 16.5\% or 6.6 billion planets of Sun-like stars have Earth-like planets with sizes within $25 \%$ of Earth's radius [5]. This value will be used in the estimates. Larger planets may acquire massive atmospheres like the Solar System's outer planets that would prohibit advanced life forms.
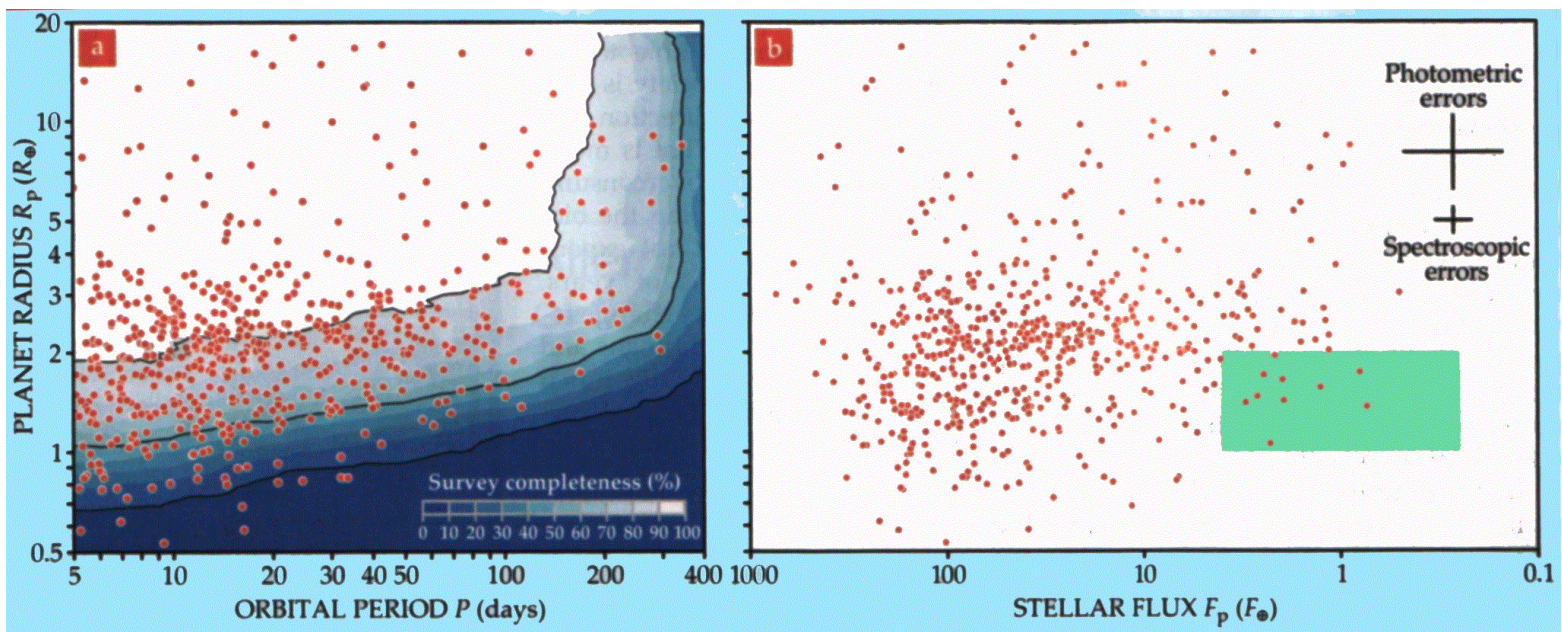

Figure 2(b): The 603 exoplanets transiting Sun-like stars found in a search of Kepler telescope data are plotted against planet radius and (a) orbital period or (b) stellar irradiation flux. In panel (a), the color scale indicates the variation of survey incompleteness $\mathrm{C}$, the fraction of planet transits in the noisy Kepler data that the search was expected to reveal. The green patch in panel (b) indicates an Earth-like region with planet radii 1-2 Earth radii and stellar fluxes within a factor of 4 of irradiation flux. The representative crosses show how uncertainties are reduced by follow-up stellar spectroscopy [1].

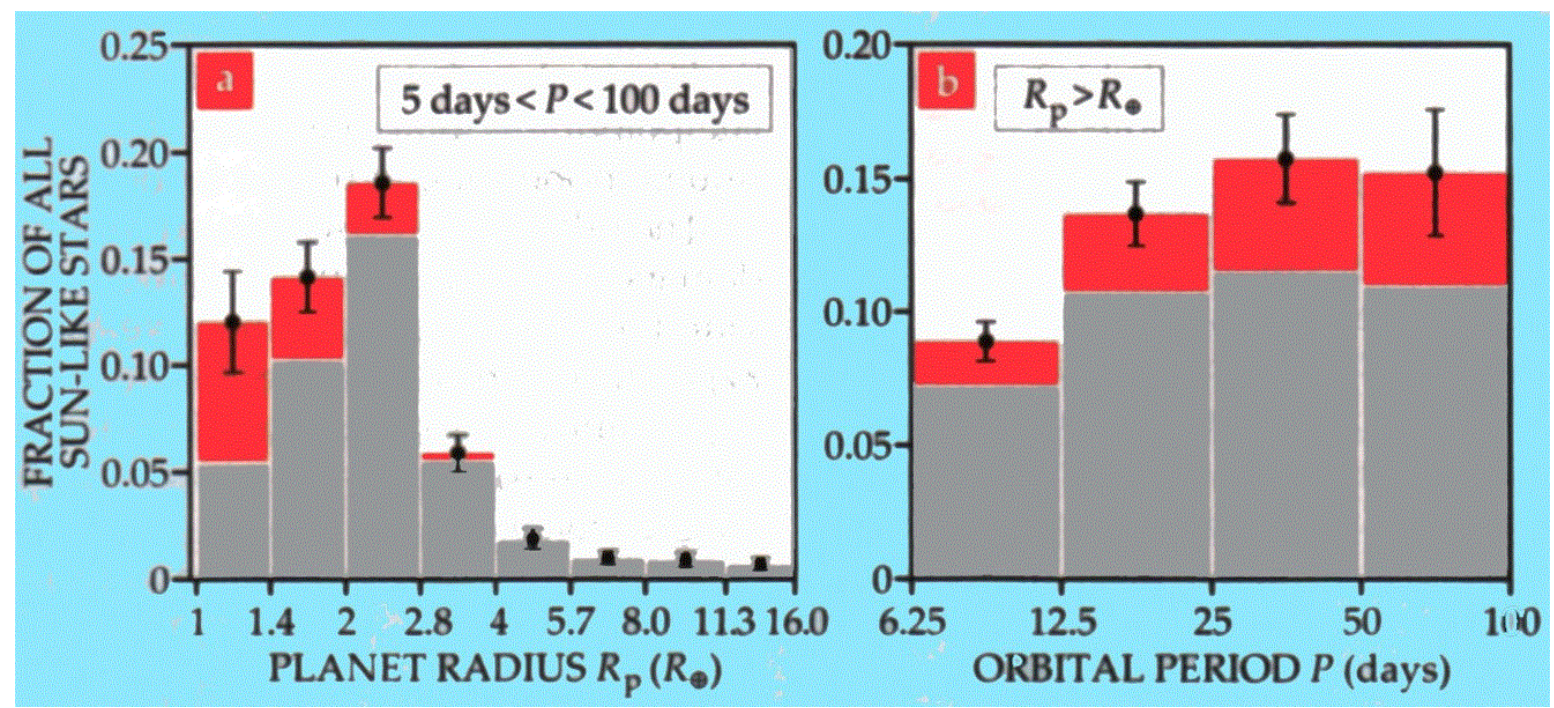

Figure 2(c): Estimated fraction of Sun-like stars having planets of different (a) sizes and (b) orbital periods P. Intervals are logarithmically equal. Gray bars represent transiting planets actually found. Red extensions are corrections for the fraction (1-C) lost in the noisy photometric data. Abundances are also corrected for all the exoplanets that do not happen to exhibit transits in Earth's direction [1].

\section{Red dwarf stars and their planets}

Red-dwarf stars comprise about $70 \%$ of the stars in our Milky Way galaxy [6]. Figure $2 \mathrm{~d}$ is a plot of the number of all-stars within 30 lightyears of the Sun as a function of stellar masses. The number of red- dwarfs below about 0.5 solar masses increases very rapidly. Since the number of stars in the galaxy is about 300 billion, then there are about 210 billion red- dwarf stars. 
Citation: Strom, Robert G (2015) We are not alone: Extraterrestrial Technological Life in our Galaxy. Astrobiol Outreach 3: 144. doi: $10.4172 / 2332-2519.1000144$

Page 5 of 18

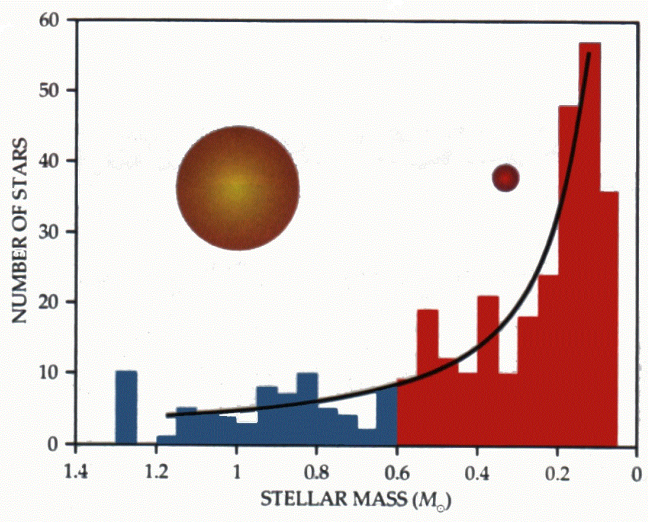

Figure 2(d): The mass distribution of all stars within 30 light-years of the Sun is plotted in units of the Sun's mass (M). The red-dwarf stars (Red), lighter than $0.6 \mathrm{M}$, are much more numerous than all heavier stellar types (Blue). The distribution rises steeply toward smaller masses. The spherical discs show the relative sizes of the Sun (left) and a typical red-dwarf (right).

The habitable zone of a red-dwarf star is much closer than a Sunlike star because the luminosity is much less. A red-dwarf with $1 / 4$ the Sun's mass will only have about $0.5 \%$ of the Sun's luminosity. Figure $2 \mathrm{e}$ is a comparison of the habitable zones around the Sun-like star 55 Cancri and that of the red-dwarf GJ 1214. The white circles show the orbits of three known planets of 55 Cancri. The two inner planets are not in the habitable zone and the outer planet has an orbital eccentricity that causes it to be partly in the habitable zone and partly in inhabitable zone. The red-dwarf GJ 1214 has luminosity less than $1 \%$ of 55 Cancri's, and a habitable zone much smaller and closer in. This red-dwarf has a planet (not shown) so close to it that it is in the inhabitable zone.

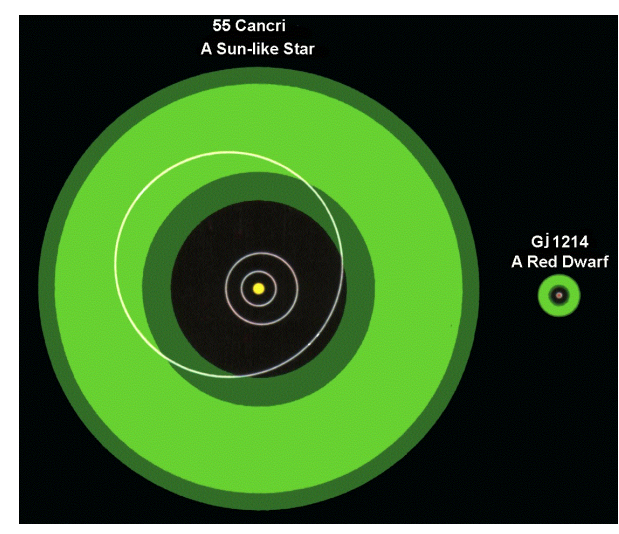

Figure 2(e): The light and dark green shading indicate conservative and permissive habitability criteria for the Sun-like star 55 Cancri and the red-dwarf GJ 1214 [6].

Recently a planet similar to the Earth in size ( $~ 10 \%$ larger) has been discovered orbiting a red-dwarf star in the habitable zone where water is stable at the surface [7]. It is called Kepler $186 \mathrm{f}$ and is about 500 light years from Earth. Kepler 186f has an orbital period of 130 days or 0.36 of an Earth year. Figure $2 \mathrm{f}$ shows the habitable zone and the orbits of the planets associated with Kepler 186.

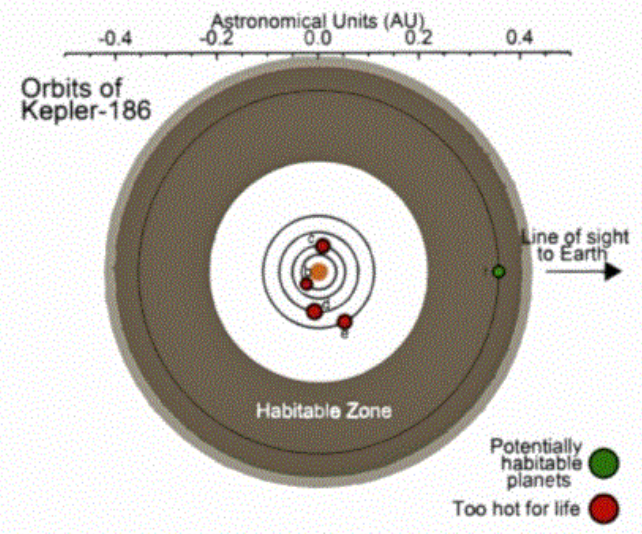

Figure 2(f): A schematic diagram of the Kepler-186 system showing the orbits of the planets. Planet $\mathrm{f}$ (green) is an Earth-sized planet in the habitable zone. The relative planet sizes are correct, but are not the same scale as the orbits that are shown in black [7].

A new study by Dressing and Charbonneau [8] using Kepler reddwarf data has measured the abundance of Earth-like planets in the habitable zones of red-dwarfs. Figure $2 \mathrm{~g}$ is a plot of the size distribution of planets (in units of Earth radii) orbiting red-dwarf stars with orbital periods less than 150 days. They far outnumber Neptunesized planets (about 4 Earth radii). This indicates there are about 0.5 Earth-sized planets per red-dwarf habitable zone.

Since there are about 210 billion red-dwarfs in the galaxy, then the 0.5 Earth-sized planets per red-dwarf habitable zone means that there are about 105 billion Earth-sized planets associated with only red$d$ warfs. That would make over 100 billion Earth-like planets in habitable zones around Sun-like and red-dwarf stars in our galaxy.

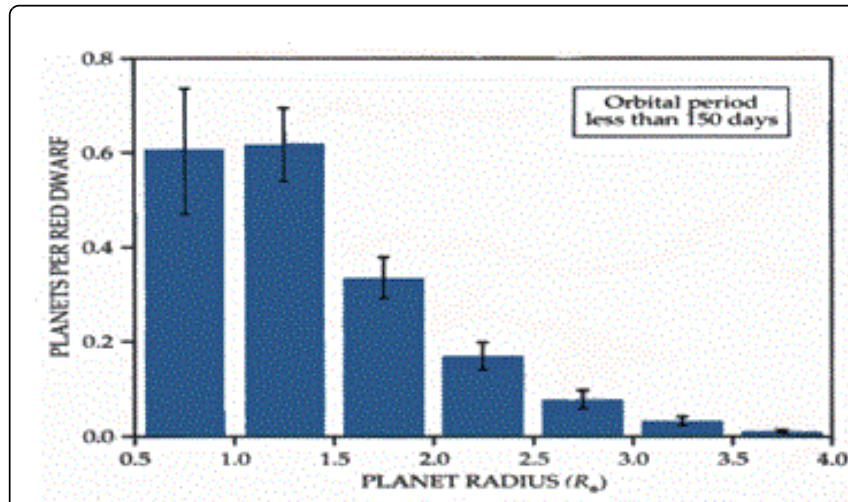

Figure 2(g): The size distribution of planets (in units of Earth radii) orbiting red dwarf stars with periods less than 150 days, as found by NASA's Kepler telescope. Earth-sized planets far outnumber Neptune-sized planets [8].

A major problem with planets around red dwarf stars is that these small stars tend to be more active with greater bombardment of solar 
flares and radiation that may be potentially harmful to any life and may prohibit its formation. It was also thought that their close proximity to the star could result in a tidal lock where only one side faces the star. This would result in a very cold night-side and a very hot dayside that could prohibit advance life forms from evolving. However, a recent study [9] showed that the atmospheric tide of planets with a relatively thin atmosphere would prevent a synchronous rotation. Therefore, Earth-like planets with about a 1 bar atmosphere orbiting stars more massive than $\sim 0.5$ to 0.7 solar mass would have nonsynchronous rotations.

Because red dwarfs are so dim and cool, a planet must exist in very close proximity to it in order to obtain adequate energy to harbor life. A planet would have to be much nearer than the Earth is to the Sun, nearer even than Mercury. This would subject it to extreme continuous flow of charged particles which streams from the star in all directions. The intense stellar wind at close distances is capable of stripping surrounding planets of their atmospheres.

The Earth very rarely feels the impact of the solar wind due to its strong magnetic field. This invisible field envelops the Earth, deflecting charged particles away from it (Figure 3L).

A recent study [10] indicated that the close proximity would strip the planets of red dwarf stars of their atmospheres and consequently their water supply over time, even with a magnetic field as strong as the Earth's. Planets in the habitable zone of red dwarfs are expected to experience intense Extreme Ultraviolet and X-ray radiation (Figure 2h). Although the environment of Red Dwarf planets would be too hostile to sustain human-type life, extremophiles (hardy organisms capable of thriving in extremely hostile environments) could possibly survive.

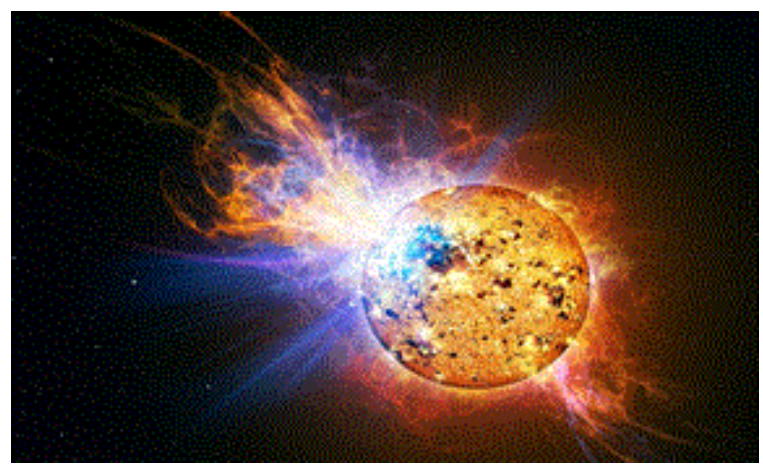

Figure 2(h): An artist's depiction of the incredibly powerful flare that erupted from a red dwarf star in 2008. Credit: Casey Reed/ NASA.

This study will consider only the $\sim 6.6$ billion Earth-like planets in the habitable regions of Sun-like stars. There are too many uncertainties with Red Dwarf planets in their habitable zones to try to estimate how many may contain intelligent life. However, using only Sun-like stars is a very conservative estimate and the reader should realize that there could be many habitable planets with an atmosphere, water, and life forms capable of withstanding the intense radiation of red dwarf stars. Even with these restrictions it will become clear that there could be thousands of planets with intelligent life forms.

\section{Part 3: Characteristics of the terrestrial planets and outer planet satellites}

The knowledge of our solar system is the only data we have about the constitution of a solar system. In particular, how characteristic the terrestrial planets are of other terrestrial-like planets in the Milky Way galaxy is completely unknown. We only have one example, which is no statistics at all. However, our terrestrial planets can be used to set some limits on the probability of other technological life in our galaxy and the Universe in general.

Our solar system consists of four terrestrial planets, an asteroid belt, and four large gaseous outer planets. There are several dwarf planets, e.g., Pluto and asteroids Ceres and Vesta, the Kuiper Belt of icy objects and the Oort cloud of comets (Figures $3 \mathrm{a}$ and $3 \mathrm{~b}$ ). There are also a large number of satellites orbiting the planets and dwarf planets; three for the terrestrial planets and 22 for the outer planets. Figure 3a shows that when the Kuiper Belt and Oort cloud are taken into consideration our solar system and its components are very large. This is illustrated by the inset showing the outer planet orbits and the Kuiper Belt. The Kuiper Belt may extend to about 50 AU from the Sun and the Oort cloud may extend to almost 2 LY. Therefore, our Oort cloud extends about half the distance to the nearest star; Proxima Centauri at 4.24 LY. The data from the Kepler mission are not sufficient to show whether or not other solar systems have asteroids, Kuiper belts or Oort clouds.

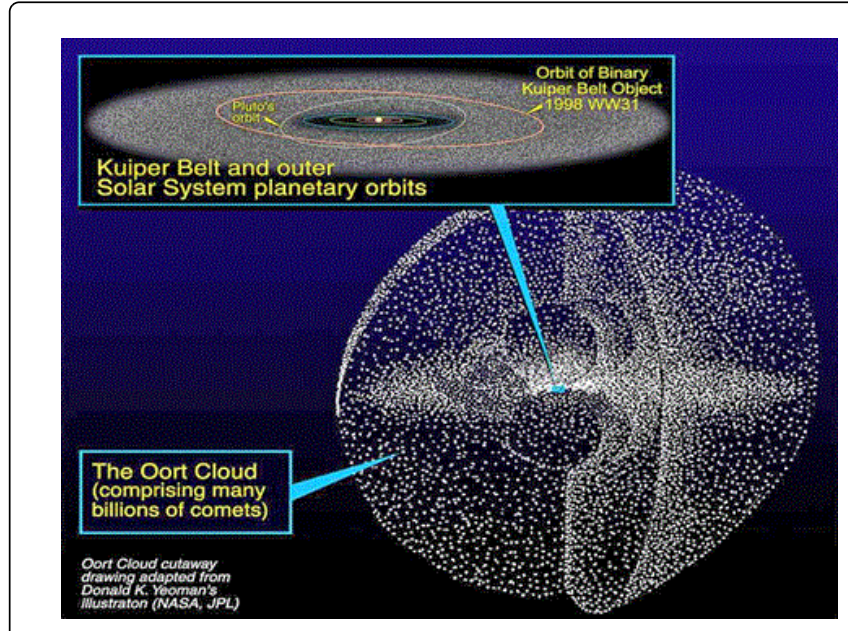

Figure 3(a): This is an artist's rendition of the Oort Comet Cloud surrounding the Kuiper Belt and outer planet orbits. On this scale the inner planet orbits are too small to show.

The characteristics and geological evolution of the terrestrial planets determine if they can accommodate liquid water on their surfaces and the length of time it will remain there. All terrestrial planets were formed 4.56 billion years ago. There are four terrestrial planets that range in radius from $2439 \mathrm{~km}$ for Mercury to $6378 \mathrm{~km}$ for Earth. Their distances range from $0.39 \mathrm{AU}$ for Mercury to 1.52 AU for Mars. Only the Earth and Mercury have magnetic fields. Table 1 lists the orbital and geometric properties of the terrestrial planets. Although Earth and Mars have similar obliquities $\left(23.4^{\circ}\right.$ and $25.2^{\circ}$ respectively) and rotation periods (1.0 days and 1.03 days respectively), Venus rotates very slowly (343 days) in a retrograde direction and Mercury rotates slowly (58.65 days) in a prograde (same as Earth) direction. 


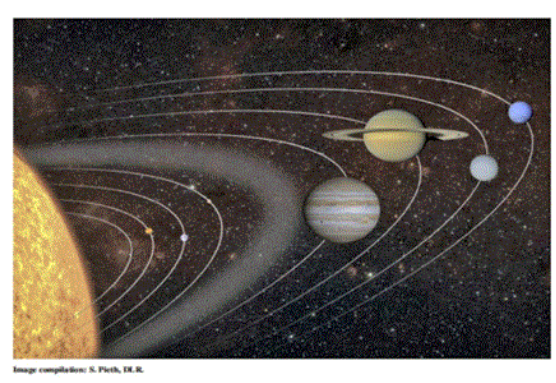

Figure 3(b): Diagram showing the orbits and size of the planets relative to the Sun. The sizes are to scale, but the distances are not to scale. The gray zone between Mars and Jupiter is the asteroid belt.

\begin{tabular}{|l|l|l|l|l|}
\hline Property & Mercury & Venus & Earth & Mars \\
\hline Distance (AU) & 0.387 & 0.723 & 1.0 & 1.524 \\
\hline Orbital Period (Days) & 87.97 & 224.7 & 365.2 & 686.98 \\
\hline Obliquity $\left(^{\circ}\right)$ & 0 & 3 & 23.4 & 25.2 \\
\hline Rotation Rate (Days) & 58.65 & $343 \mathrm{R}^{*}$ & 1.0 & 1.03 \\
\hline
\end{tabular}

Table 1: Motion and geometric properties of the terrestrial planets ${ }^{\star} \mathrm{R}=$ retrograde rotation. Obliquity is the inclination of the rotation axis to the plane of the orbit. One AU (Astronomical Unit) is the distance from the Earth to the Sun.

It is probable that some of the terrestrial planet characteristics were caused by very large planet-size impacts during the final stages of planetary accretion. The very large iron core of Mercury (84\% of the radius) may have been the result of a planet-sized impact that blew off most of the crust and mantle. Also the very slow retrograde rotation of Venus could be caused by such a large grazing impact, and the Moon may also have been caused by a large impact on Earth. The crustal dichotomy of Mars may also have been caused by a large impact. Other extra-terrestrial planets may have gone through similar episodes of very large post-accretion impacts.

All of the terrestrial planets experienced a period of Late Heavy Bombardment (LHB). It started about 4.1 Ga, peaked about 3.9 Ga, and rapidly declined in the next 100-200 million years. Some of the LHB objects continued to impact the Earth until about $2 \mathrm{Ga}$ but at a much lower rate. The LHB was caused by sweeping of resonances through the asteroid belt and the Kuiper Belt objects beyond the orbit of Neptune. The sweeping of resonances was caused by the migration of Jupiter and Saturn. The heavily cratered regions of Mercury, the Moon, and Mars were primarily the result of asteroids from the LHB [11]. That record has been erased on Venus and Earth by geologic processes. We do not know if other planetary systems have asteroid and Kuiper belt objects because they are too small to detect. However, it is likely that they at least have the equivalents of Kuiper belts since they are the remnants of planetary accretion and they probably have "Oort Clouds" of comets. We do know that some extra-terrestrial planets have migrated which may have triggered heavy bombardments on other planets in these systems.
We do not know how representative our terrestrial planets are to others in the galaxy, but they will give an idea of what is possible and some constraints on surface water and possible life.

\section{Mercury}

Mercury is the smallest planet and closest to the Sun (Figure 3c). Its distance from the Sun is $0.39 \mathrm{AU}$ and its surface conditions are completely unsuitable for life. Mercury has a weak magnetic field. It has no atmosphere, and surface temperatures range from $427^{\circ} \mathrm{C}$ on the sun-lit side to $-183^{\circ} \mathrm{C}$ on the night side. It is definitely not in the habitable zone and surely did not have any liquid water on its surface.

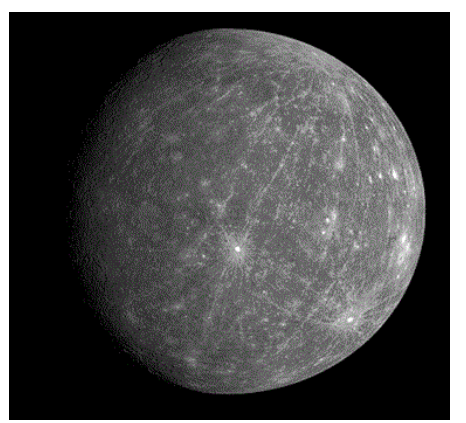

Figure 3(c): This image mosaic of Mercury was taken by the MESSENGER spacecraft and shows a cratered surface similar the Moon. It has no atmosphere and varies from extremely hot on the sun-lit side to extremely cold on the night side.

\section{Venus}

Venus (6051 km radius) is about the same size as Earth $(6378 \mathrm{~km}$ radius). Figure $3 \mathrm{~d}$ shows the atmosphere, and Figure $3 \mathrm{e}$ is a radar surface image-mosaic from the Magellan Mission. Its distance of 0.72 $\mathrm{AU}$ has been considered to be outside the habitable zone, largely because it has an atmosphere of about $\sim 96 \% \mathrm{CO}_{2}$, a surface temperature of $480^{\circ} \mathrm{C}$, and a surface pressure of 92 bars, or 92 times the Earth's atmospheric surface pressure. However, these conditions are largely due to its geologic history rather than its current position in the Solar System. Venus has a very slow retrograde rotation (343 days) and no magnetic field.

Its current surface shows no signs of plate tectonics, and its topography is very different from that of Earth (Figures $3 \mathrm{f}$ and $3 \mathrm{~g}$ ). On Earth the elevations are bimodal representing the ocean floors and continents. However, on Venus the distribution is unimodal with the mean elevations peaking at about the equivalent of $3 \mathrm{~km}$ on Earth.

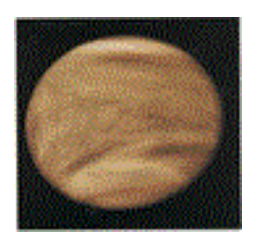

Figure 3(d): This image of Venus' atmosphere was taken in ultra violet wavelengths. It shows the structure of this 92 bar $\mathrm{CO}_{2}$ atmosphere that makes the surface temperature about $480^{\circ} \mathrm{C}$. 
Citation: Strom, Robert G (2015) We are not alone: Extraterrestrial Technological Life in our Galaxy. Astrobiol Outreach 3: 144. doi: $10.4172 / 2332-2519.1000144$

Page 8 of 18

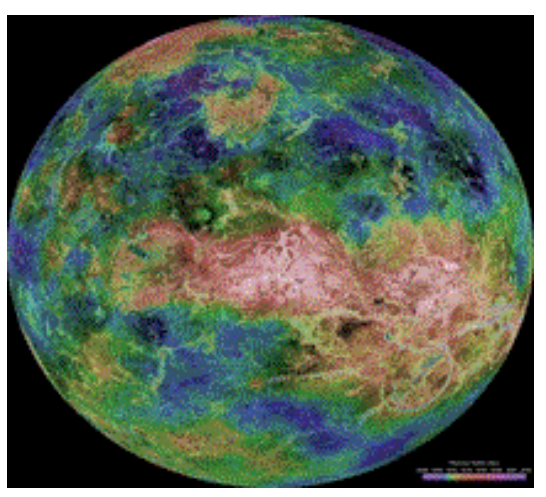

Figure 3(e): This is a Magellan radar image mosaic of the surface of Venus that is totally different from the other solid bodies in the Solar System. The whitish and reddish areas are high and the blue and green areas are low.
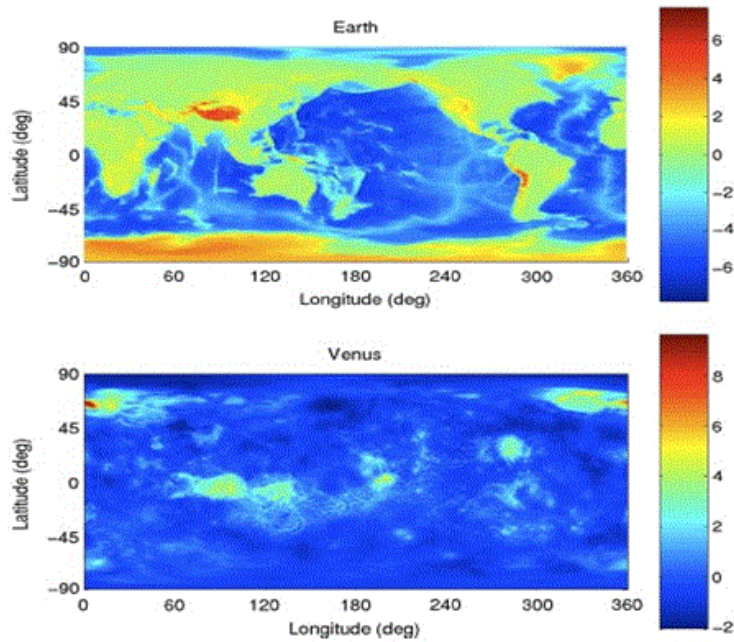

Figure 3(f): Comparison of the topography of the Earth and Venus. The color scale on the right is the elevation in kilometers. Most of Venus is at an elevation similar to the Earth's ocean basins (Figure 3g). From Smrekar SE, ER Stofan and N Mueller, Venus:Surface and Interior, Encyclopedia of the Solar System, 3rd Edition, 2015.

This main elevation primarily represents the low volcanically flooded regions on the planet. The highest areas are primarily shield volcanoes and tectonic uplifts.

Venus has a unique surface history. It is the only planet or large satellite that has a completely random impact crater distribution (Figures $3 \mathrm{~h}$ and i). This and other evidence indicates that Venus experienced multiple periods of global resurfacing mainly due to massive volcanism [12]. Fully $84 \%$ of the craters are in pristine condition and only $12 \%$ are fractured. Monte Carlo simulations indicate that only $4-6 \%$ of the planet has been volcanically resurfaced since the last global event. Some minor volcanism may still be active today.

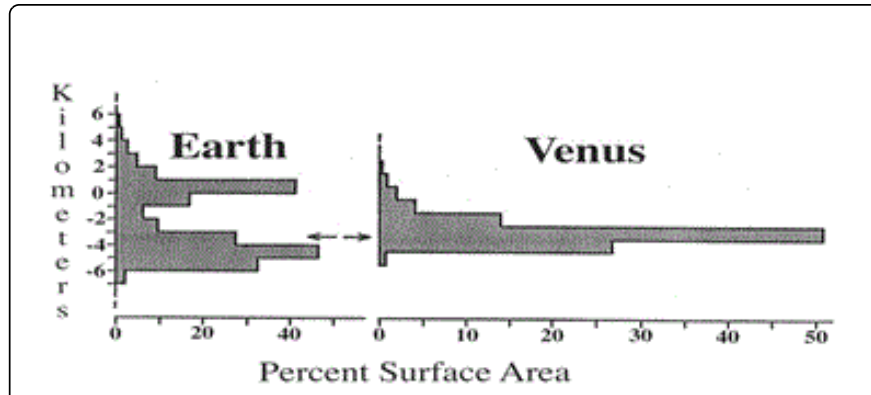

Figure 3(g): This diagram shows the distribution of elevations on Earth and Venus. The Earth has two main distributions of elevations, one representing the ocean floor and the other the continental regions. The arrows indicate the mean elevation of Earth and Venus.

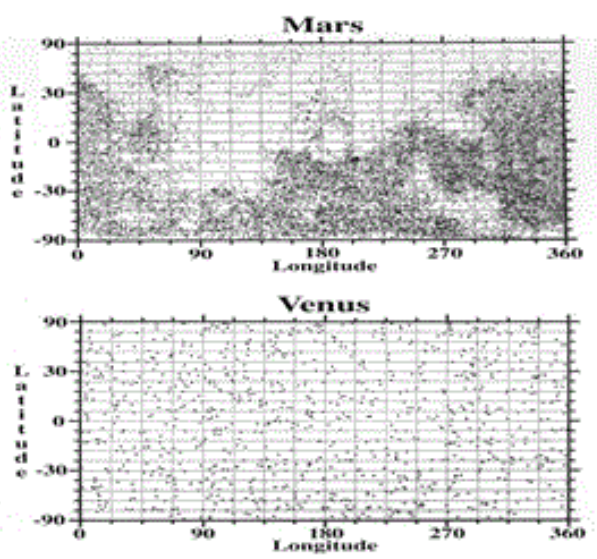

Figure 3(h): These diagrams are the distribution of craters on Mars and Venus. On Mars the crater distribution is very non-random and on Venus it is totally random.

A thermal model [13] explains how a stress-free deformable lithosphere unique to Venus is easily incorporated in the mantle to cause global resurfacing followed by cooling of the crust. This can occur multiple times. Based on its crater density, the last global resurfacing event was about 240 million years ago. These massive volcanic resurfacing periods must have emitted huge amounts of $\mathrm{CO}_{2}$ causing the 62 bar atmosphere. Currently the highly reflective clouds reflect most of the Sun's radiation back to space. It is the greenhouse effect of the massive $\mathrm{CO}_{2}$ atmosphere that causes the high surface temperature. Therefore, it is not Venus' current position that causes its very hot temperatures; it is Venus' geologic history. If Venus were at the Earth's distance from the Sun its geologic history would still produce large amounts of $\mathrm{CO}_{2}$, it's very high temperatures, and the current lack of water and habitability. This demonstrates that an Earth-sized planet in the habitable zone need not have surface water or life. 


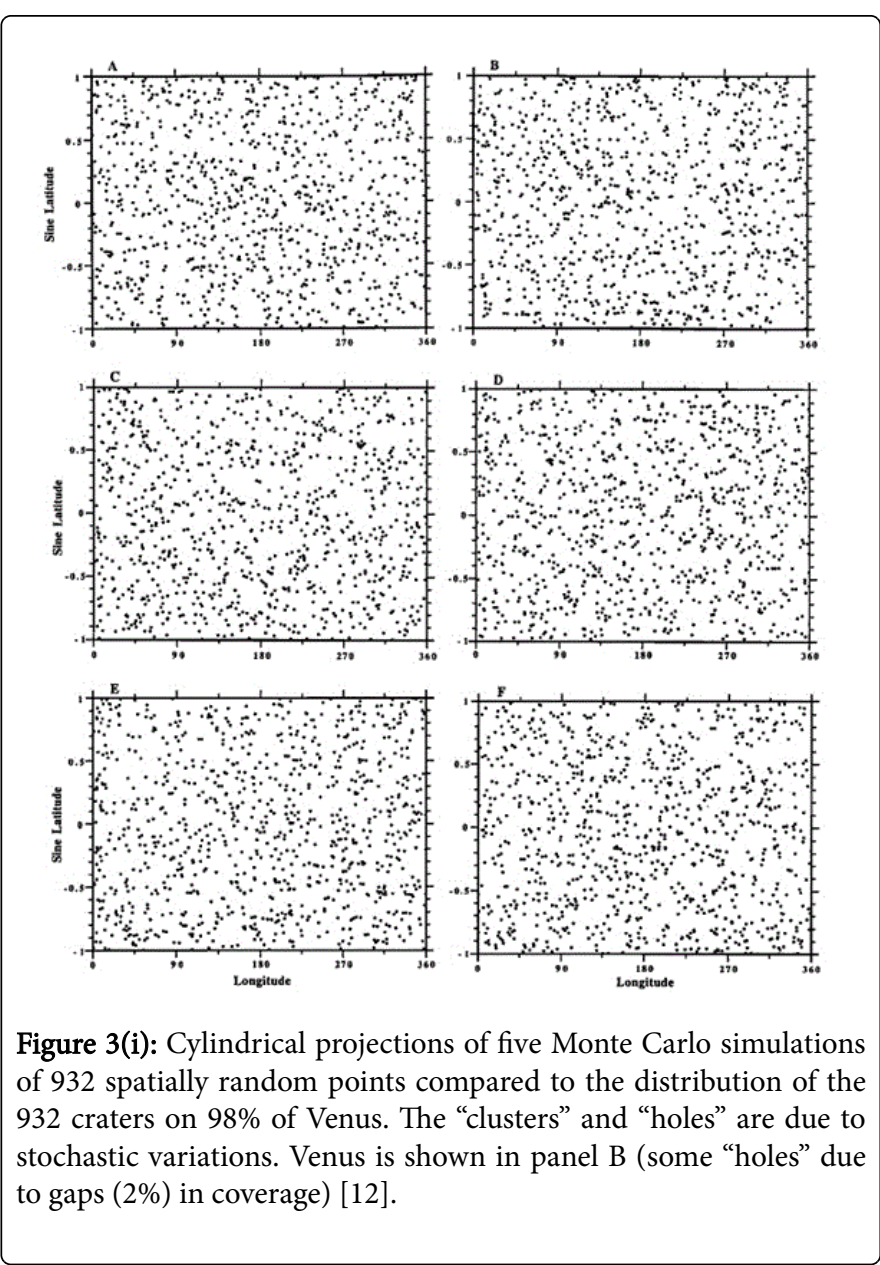

\section{Mars}

Mars is near the outer limit of the habitable zone at a distance of $1.52 \mathrm{AU}$ (Figures $3 \mathrm{j}$ and $\mathrm{k}$ ). Its diameter is $6792 \mathrm{~km}$ or $53 \%$ of the Earth's diameter. It currently does not have a magnetic field, but there is evidence that it once had one over 2 billion years ago. Mars' rotation period (1.03 days) is similar to Earth's and its current axial inclination $\left(24^{\circ}\right)$ is also similar to Earth's. There is no evidence that it experienced plate tectonics. Its atmosphere is about $95 \% \mathrm{CO}_{2}$ with a surface pressure of only 0.007 bar. Because of the low pressure the greenhouse effect actually cools the atmosphere as it does in the Earth's stratosphere with the same pressure.

There is now abundant evidence that Mars once had a much thicker atmosphere, an ocean in the northern plains and lakes in many of its impact craters. There are also complex dendritic channels indicative of running water, and large outflow channels probably due to catastrophic floods. Orbital radar data indicates that many of what were interpreted as debris flows are, in fact, debris covered glaciers, indicating that it once snowed on Mars. The water vapor responsible for the snow was probably from the ocean and crater lakes. The estimated age of the ocean and glaciers is from 3.1 to 3.5 billion years ago. Today there is no surface water. Mars probably lost most of its atmosphere about 3 billion years ago due to loss of its magnetic field and the impact of solar energetic particle bombardment that eventually striped Mars of its much denser atmosphere.
There may be signs of primitive life forms in carbon compounds discovered by the Curiosity rover, but that is not yet conclusive. In any event, if life began on a young Mars it did not evolve into complex forms. However, if life did evolve on Mars then it is very likely that life will develop on planets with surface water. Therefore, the likelihood of life on planets with surface water in the habitable zone should be relatively high if future missions detect organic life forms on Mars.

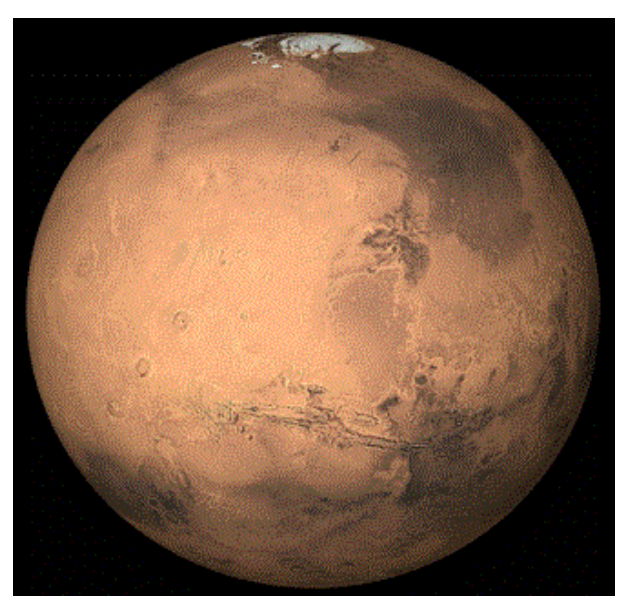

Figure 3(j): This image of Mars shows the Valles Marinaris and the string of 3 large shield volcanoes.

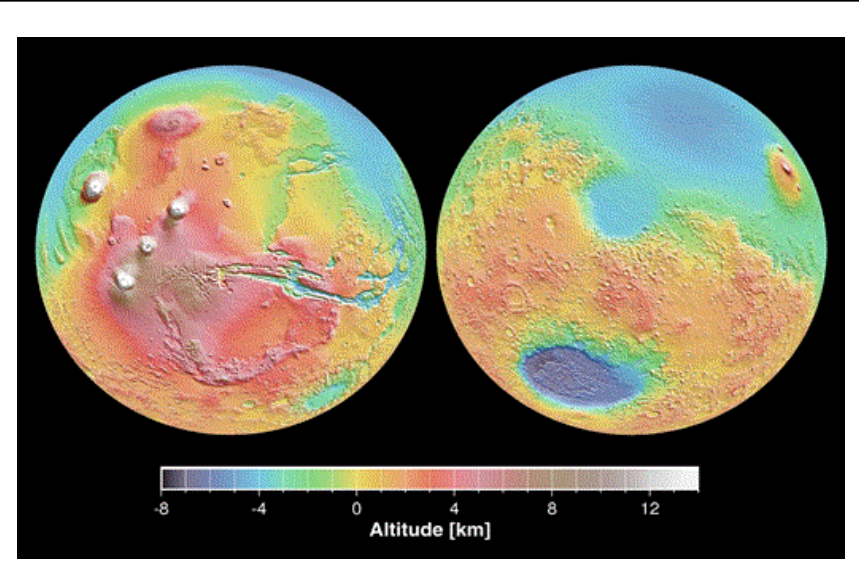

Figure 3(k): This topographic map of Mars shows the low areas in blue and the higher areas in orange and red. The four white areas are very large volcanoes. The blue area in the northern hemisphere was once the site of an ocean.

\section{Earth}

Earth has a strong magnetic field (Figure 31), and a long history of plate tectonics dating back to Precambrian times. It is at a distance of 1 AU from the Sun. Earth and Venus are about the same size (Figure $3 \mathrm{~m}$ ), but their surfaces are totally different because Venus had an entirely different geological evolution as discussed earlier. Earth's oceans comprise about $70 \%$ of the surface (Figure 3n).

The origin of Earth's water has been attributed to the Late Heavy Bombardment (LHB) by comets and water-rich asteroids about 3.9-4.1 
billion years ago. However, there is evidence from pre-Cambrian zircons that Earth had oceans as early as 4.3 billion years ago, or at least 200 million years before the LHB [14]. This would indicate the water was present well before the LHB and was intrinsic to Earth. Furthermore, a recent study [15] of deuterium-to-hydrogen enrichments finds that the solar nebula had abundant water ice that was available for comet and planetary water formation. Recent studies of Comet 67P/Churyumov-Gerasimenko by the ESA Rosetta mission indicate that its water is much richer in deuterium than Earth's water. This seems to indicate that comets are not the source of Earth's water. In this study we consider Earth's water to be derived primarily from the solar nebula, but additions probably occurred from comets and waterrich asteroids.

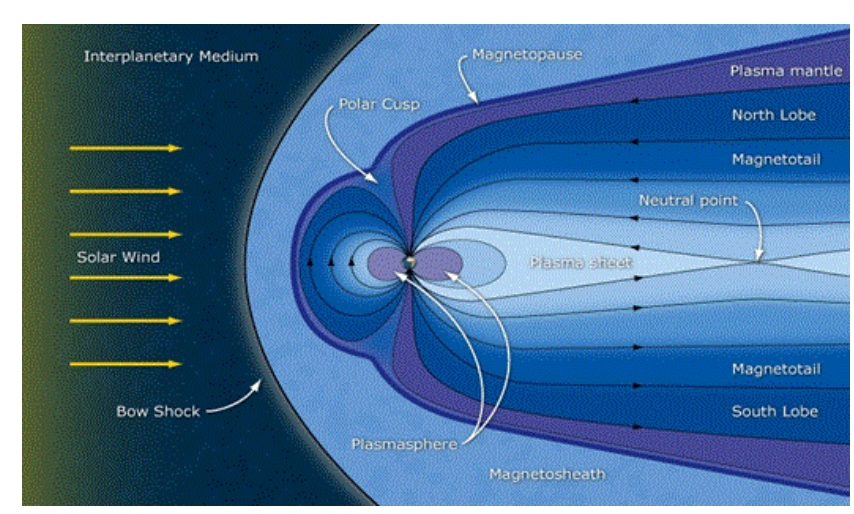

Figure 3(l): This is a diagrammatic representation of Earth's magnetic field. It has protected the Earth from the Sun's solar wind and charged particle bombardment.

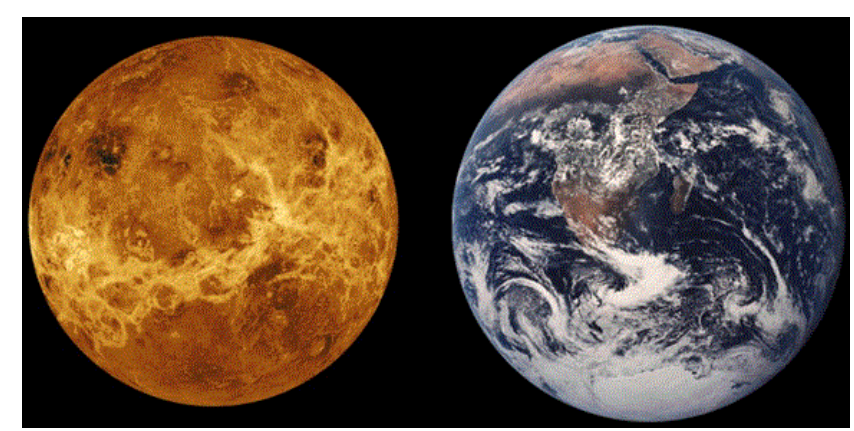

Figure 3(m): Size and surface comparison of Venus and Earth.

Life probably evolved in the early oceans from compounds of the six essential elements required of all life; carbon, hydrogen, oxygen, phosphorus, sulfur and nitrogen. We do not know how life originated from these non-organic compounds, but the energy was probably provided by photosynthesis. The earliest most primitive life on Earth was prokaryotes (unicellular organisms whose cells lack a membranebound nucleus). They were present about 3.6 billion years ago (Ga). Life may have occurred earlier, but that record has not yet been found. The first complex life (cyanobacteria using photosynthesis) began about $3.5 \mathrm{Ga}$. Complex cells (eukaryotes) began about $2 \mathrm{Ga}$ and multicellular life started about $1 \mathrm{Ga}$. Complex animals (animals with a back and front) began about $550 \mathrm{Ma}$. From this time evolution happened rapidly during the last $12 \%$ of Earth history. Land plants began about 475 million years ago, and amphibians about 360 million years ago with the first migration and adaption to land. Reptiles began about 300 million years ago, and mammals about 200 million years ago.

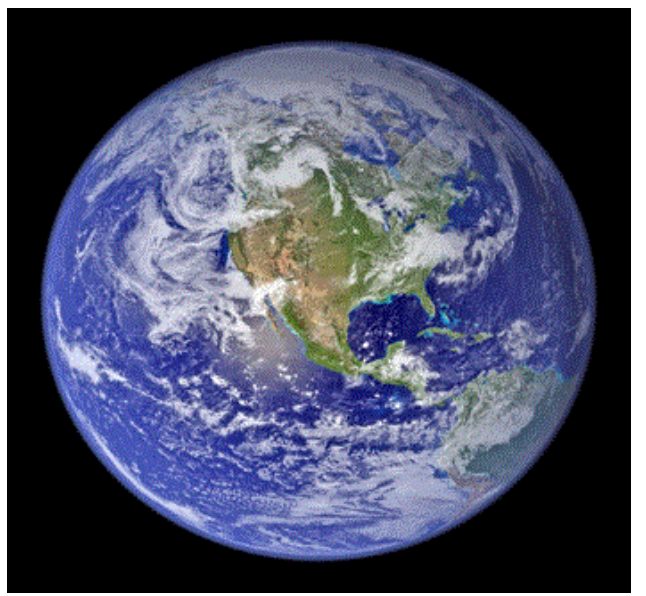

Figure 3(n): This image of the Earth shows the North American continent, the cloud cover and the blue oceans. The oceans cover about $70 \%$ of the Earth's surface. The Earth is totally different from the other solid bodies in the Solar System.

There have been 5 mass extinctions where greater than $50 \%$ of species became extinct. At least two were caused by climate changes. The largest mass extinction was the Permian/Triassic extinction that caused $\sim 95 \%$ of the species to perish about 252 million years ago. It was caused be the massive eruption of the Siberian flood basalts that covered an area about the size of the United States. The eruptions resulted in the release of massive amounts of greenhouse gases that drastically warmed the planet and drastically acidified the oceans over a period of about 10,000 years.

The mass extinction at the Cretaceous/Tertiary boundary (K/T extinction) 66 million years ago was also caused by a climate change that killed $76 \%$ of all species including the non- avian dinosaurs. This extinction was probably caused by a large impact of a $\sim 10 \mathrm{~km}$ asteroid that formed the $180 \mathrm{~km}$ diameter Chicxulub crater on the Yucatan peninsula of Mexico. The impact probably triggered the enormous Wai Subgroup of Deccan flood basalt lava flows, which may account for $>70 \%$ of the Deccan Traps main-stage eruptions [16]. This eruption of the Deccan flood basalts is the same age at the Chicxulub impact [17], and released massive amount of $\mathrm{CO}_{2}$. This mass extinction of $76 \%$ of all species was a result of a rapid extreme climate change caused by both events. However, it was probably due mostly to the impact because the Earth was already in a Hot House, and the size of the Deccan eruptions $(500,000 \mathrm{~km} 2)$ was considerably less than the Siberian eruptions (2-7 million $\mathrm{km} 2$ ) that caused the Permian/Triassic mass extinction. Furthermore, the impact first caused warming, and then a major cooling for about 10 years with temperatures equivalent to an Ice Age.

During the impact there was first an extreme heating of the atmosphere by the entry of impact ejecta. This was followed by freezing conditions for about 10 years from huge amounts of atmospheric aerosols ejected into the stratosphere that reflected sunlight back to 
Page 11 of 18

space. At that time the planet was in what is called a "Hot House" with global average temperatures about $9^{\circ} \mathrm{C}$ warmer than today. The climate change from very hot to ice age temperatures resulted in the extinction of the non-avian dinosaurs, but some mammals survived because they were burrowing animals that largely avoided the fatal effects of extreme climate change. Furthermore, their greatest predator, the dinosaurs, had been eliminated by the climate change. After the demise of the dinosaurs, the mammals flourished in the Cenozoic era known as the "Age of Mammals". If that extinction had not happened dinosaurs would still be here devouring mammals and keeping them in a relatively primitive state, and almost certainly we would not be here today. We owe our existence to the K/T extinction that occurred 66 million years ago.

A new study indicates that Earth has entered its 6th mass extinction event [18]. These results are consistent with earlier studies by Duke University and E. Kolbert's book titled "The Sixth Extinction". The new study found that vertebrates are disappearing at a rate 114 times faster than when Earth was not going through a mass extinction. It could be 1000 times faster. From 1900 to present, more than 400 vertebrate species have become extinct. Normally this type of loss would take about 10,000 years. About $41 \%$ of amphibians and $25 \%$ of mammals face extinction. According to the lead author of this new report, "If it is allowed to continue, life would take many millions of years to recover and our species itself would likely disappear early on". The current extinctions are the result of climate change, deforestation, pollution, and over population.

Below are two tables where the geologic timescale and human evolution are shown relative to one year. This puts in perspective our species evolution compared to the geologic timescale.

We know less about the Precambrian Eon, which comprises $88 \%$ of geologic time, than any other time in geologic history. On the geologic time scale relative to one year it lasted 10 months and 17 days from Jan. 1 to Nov. 18. The Cenozoic Era that started after the 5th mass extinction and includes human evolution comprises only $1.4 \%$ of geologic time. Relative to one year it started on Dec. 26th and has duration of only 5 days. Human history (Homogenus) began $\sim 2.8$ million years ago, or the early evening of the last day of the year on a time scale relative to 1 year (Table 3 ).

\begin{tabular}{|l|l|l|l|l|}
\hline \multirow{2}{*}{ Eon or } & \multicolumn{2}{|l|}{ Absolute Time } & \multicolumn{2}{l|}{ Relative to a Year } \\
\cline { 2 - 5 } & $\begin{array}{l}\text { Duration } \\
\text { (million } \\
\text { years) }\end{array}$ & $\begin{array}{l}\text { Percent } \\
\text { Geologic } \\
\text { Time }\end{array}$ & $\begin{array}{l}\text { Starting } \\
\text { Time }\end{array}$ & Duration \\
\hline Precambrian & 3,996 & 88.0 & Jan. 1 & 10 mo. 17 days \\
\hline Paleozoic & 296 & 6.5 & Nov. 18 & 24 days \\
\hline Mesozoic & 183 & 4.1 & Dec. 12 & 15 days \\
\hline Cenozoic & 66 & 1.4 & Dec. 26 & 5 days \\
\hline
\end{tabular}

Table 2: The geologic timescale relative to the duration of one year.

It is important to understand that our technology occurred very late on the geologic time scale and that other present technological civilizations in our galaxy probably developed much earlier than ours. Therefore, they should be much more advanced than our technology. On a geologic time scale relative to one year, our technology began 1.7 seconds ago and our manned exploration of space began only about 0.2 seconds ago (Table 3 ).

\begin{tabular}{|c|c|c|c|}
\hline \multirow[t]{2}{*}{ Major Event } & \multirow{2}{*}{$\begin{array}{l}\text { Years Before } \\
\text { Present }\end{array}$} & \multicolumn{2}{|c|}{ December 31} \\
\hline & & Starting Time & Duration* \\
\hline Human Development Begins & $\sim 7$ million & $10: 30 \mathrm{AM}$ & $13 \mathrm{hrs}, 30 \mathrm{~min}$. \\
\hline Homo Genus Begins & $\sim 2.8$ million & 6:30 PM & $5 \mathrm{hrs}, 30 \mathrm{~min}$ \\
\hline Early Homo Sapiens & 400,000 & $11: 14$ PM & $46 \mathrm{~min}$. \\
\hline Homo Sapiens Sapiens (us) & 125,000 & 11:46 PM & $14 \mathrm{~min}$. \\
\hline Civilization Begins (Agriculture) & 12,000 & 11:58:21 PM & $1 \mathrm{~min} .21 \mathrm{sec}$. \\
\hline Bronze Age Begins & 5000 & 11:59:34 PM & $35 \mathrm{sec}$. \\
\hline Early Writing & 4000 & 11:59:32 PM & $28 \mathrm{sec}$. \\
\hline Industrial Revolution (Technology) & 250 & 11:59:58.3 PM & $1.7 \mathrm{sec}$. \\
\hline Radio Transmission Begins ${ }^{1}$ & 92 & 11:59:58.9 PM & $0.6 \mathrm{sec}$. \\
\hline Human Lifetime & $\sim 75$ & 11:59:59.7 PM & $0.3 \mathrm{sec}$ \\
\hline Current Rapid Global Warming & 25 & 11:59:59.9 PM & $0.1 \mathrm{sec}$. \\
\hline
\end{tabular}

Table 3: The evolution of humans on a geologic timescale relative to one year as in Table 2. On this relative timescale all human evolution starts on the morning of Dec. 31.

${ }^{*}$ Starting Time to Present. 
Human evolution began about 7 million years ago, and our species began about 125,000 years ago. Civilization began about 12,000 years ago and the Industrial Revolution (Technology) began only 250 years ago. We began radio transmission that would have been emitted to the rest of the Universe only 92 years ago. That signal of our existence has now traveled only $92 \mathrm{LY}$ into our galaxy (Figure 1f). Since our galaxy is about $110,000 \mathrm{LY}$ in diameter, our signal has only traveled $0.0008 \%$ of the galactic diameter.

On Earth it took 4.56 billion years for humans to evolve and develop technology. During this time 99.9\% of all species that ever inhabited the Earth became extinct. We are here because a mass extinction killed the dinosaurs and allowed us to evolve from the mammals that flourished after the impact. Will we be the exception and escape extinction, or will we also become extinct like the vast majority of other species? Currently Earth apparently has entered the 6th mass extinction due to climate change, deforestation, pollution and over population. Unless we do something to stop these conditions soon, it is likely we will become extinct in the not too distant future.

\section{Other Water/Ice Bodies in the Solar System}

There is a good possibility that some of the icy outer planet satellites, including Jupiter's Europa and Ganymede, and Saturn's Enceladus, have subsurface water layers. It is also possible that life could have originated in these subsurface "oceans" by chemoautotrophism where energy is obtained by oxidation of inorganic substances such as ferrous iron, ammonia, molecular hydrogen, hydrogen sulfide and others. In this case photosynthesis, where energy is acquired from light, is not required. However, it is highly unlikely that life in these circumstances would evolve into intelligent technological beings able to communicate with other worlds. This type of life will not be discussed in this paper.

\section{Part 4: Life and technological beings on other earth-like planets}

A technological society consists of intelligent beings that have developed machinery or complex devices that facilitate productivity. Our technological society began with the Industrial Revolution in about 1760. It included going from hand production methods to machines, new chemical manufacturing and iron production processes, improved efficiency of waterpower, the increasing use of steam power and the development of machine tools. It began in Great Britain and within a few decades had spread to Western Europe and the US. However, this beginning of technology did not produce any communications that could be detected by other beings in some other part of the galaxy. That began in 1922 when the BBC began broadcasting radio programs to a wide audience. On a geologic time scale relative to 1 year (Table 3 ) it would have happened only 0.6 seconds ago. Therefore, we have only been a technological society able to make ourselves known to another extra-terrestrial society for the past 92 years with radio waves. Consequently, our presence could only be detected by radio waves within a radius of $92 \mathrm{LY}$ of our Sun or less than $99.9 \%$ of the galaxy. However, if they could detect street lighting on our planet they may have detected us 135 years ago (Part 6).

\section{The search for extraterrestrial intelligence (SETI)}

The SETI (Search for Extra-Terrestrial Intelligence) project has for the past three decades been conducting a search for signals being transmitted by extraterrestrial life located outside the Solar System, primarily in the radio frequencies of the electromagnetic spectrum. These searches are directed at specific stars and to date the project has not detected any signal. Some of the most well-known projects are run by Harvard University, the University of California, Berkeley, and the SETI Institute.

SETI searches for electromagnetic radiation emitted by advanced technologies. Many radio frequencies penetrate our atmosphere, and this led to radio telescopes that investigate the cosmos using large radio antennas. Human activities emit considerable electromagnetic radiation as a byproduct of communications such as television and radio. These signals would be easy to recognize as artificial due to their narrow bandwidths.

There should be at least several technologies that are emitting electromagnetic radiation similar to ours. Astronomers are puzzled by the apparent absence of technological detectable signals when there are so many Earth-like planets. If we are representative, it may be that other technological beings also have very short lifetimes. Maybe we have missed signals because they have become extinct before we had the ability to detect such signals. The other question that needs to be considered is the possibility of the development of a technological society on a given Earth-like planet. It is possible that the evolution of intelligent beings is rare and that we may be the exception to the rule. Maybe these factors are at least possible reasons for the lack of detection of other technological civilizations.

Dr. Frank Drake devised an equation to determine the number of technological civilizations trying to communicate with us at the present time. It is called the Drake Equation:

\section{$\mathrm{N}=\mathrm{Rf}_{\mathrm{p}} \mathrm{n}_{\mathrm{e}} \mathrm{f}_{\mathrm{f}} \mathrm{f}_{\mathrm{i}} \mathrm{f}_{\mathrm{c}} \mathrm{L}$}

where $\mathrm{N}$ is the present number of technological civilizations trying to communicate with us, $R$ is the rate of star formation, $f_{p}$ is the probability that the new stars have planets, $n_{e}$ is the number of those planets capable of supporting life, $\mathrm{f}_{\mathrm{l}}$ is the probability that those planets will actually develop life, $f_{i}$ is the probability that life will develop intelligence, $f_{c}$ is the probability that intelligent life will develop the technology capable of interstellar communication, and $\mathrm{L}$ is length of time that communication, once started, will continue. Although these are important probabilities, there are others that should be considered.

From the extra-terrestrial planet study discussed previously, there may be $\sim 6.6$ billion Earth-like planets similar in size to Earth in the habitable zone of Sun-like stars. This number could replace $n_{e}$ as the present number of Earth-like planets in the habitable zone, but there are other important considerations (some the same as in the Drake equation) that need to be taken into account before we could say that they actually develop technological life. These are discussed below.

\section{Other questions to answer}

There are several other questions that must be answered in order to estimate the number of technological beings that may be in our galaxy:

- How many Earth-like planets have standing bodies of water on their surface?

- On how many of these planets did life originate?

- On how many of these planets did life migrate and adapt to land? This assumes that life started in a water environment.

- On how many of these planets did "intelligent" life evolve?

- On how many of these planets did technological life develop?

- If technological life developed, how many are present today? 
The Drake Equation could be rewritten as follows:

\section{$\mathrm{N}=\mathrm{n}_{\mathrm{h}} \mathrm{f}_{\mathrm{w}} \mathrm{f}_{1} \mathrm{f}_{\mathrm{ld}} \mathrm{f}_{\mathrm{i}} \mathrm{f}_{\mathrm{t}} \mathrm{f}_{\mathrm{c}}$}

where $\mathrm{N}$ is the present number of technological civilizations, $\mathrm{n}_{\mathrm{h}}$ is the number of Earth-like planets in habitable zones, $f_{w}$ is the percentage of planets that have surface water, $f_{1}$ is the percentage that have life, $f_{l d}$ is the percentage where life migrated to a land area, $f_{i}$ is the percentage where intelligent life evolved, $f_{t}$ is the percentage where technological life developed, and $\mathrm{f}_{\mathrm{c}}$ is the percentage of technological life that is currently present. Of the seven factors in the above equation only the first one $\left(\mathrm{n}_{\mathrm{h}}\right)$ is based on extrapolated observations from Kepler mission data.

These questions cannot be answered with any certainty, because Earth is the only habitable planet we know. Even Earth itself may be very unusual and not typical of other Earth-like planets. Furthermore, there are still many things we do not fully understand about our own Solar System and the history of the terrestrial planets. However, it is possible to make some educated guesses based on our current understanding of the Solar System and the occurrence and history of life on Earth.

\section{Part 5: Constraints on determining the number of technological civilizations}

Before the possible number of technological civilizations in our galaxy can be determined it is necessary to discuss the factors that determine that number. The reliability of estimates for the different factors varies greatly, from completely unknown to moderate confidence. The estimated percentages will be "optimistic", "pessimistic" and "unlikely". They will be determined for Earth-like planets between about 0.75 to 1.25 Earth radii in the habitable zones of Sun-like stars $\left[\mathrm{n}_{\mathrm{h}}=6.6\right.$ billion]. The "unlikely" value is half the "pessimistic" value. The possible 105 billion Earth-like planets in the habitable zones of Red Dwarfs will not be considered because of the problems discussed earlier. However, there could be a substantial number that could survive the harsh radiation environment of Red $D$ warf stars. Therefore, this evaluation is very conservative.

\section{Earth-like planets with standing bodies of water on their surface [fw]}

The signature of water has been observed in giant molecular clouds between the stars, in disks of material that represent newborn planetary systems, and in the atmospheres of giant planets orbiting other stars. This suggests that water may be very common in planetary systems.

We are fairly sure that water is required for life to develop. Does this water have to be on the surface of a planet? It has already been mentioned that life could originate in subsurface oceans that probably occur on outer planet satellites like Europa and Ganymede at Jupiter, and Enceladus at Saturn by chemoautotrophic processes in the absence of light. However, it is highly unlikely that this life would evolve into intelligent technological societies. It is not even certain that all planets with surface water will develop intelligent technological life.

We only know two planets in our Solar System that have had surface water. One, of course, is Earth, and the other is Mars. A very early Venus may have had surface water but it was lost early in its history. We know from its geology that Mars once had a denser atmosphere, flowing rivers and standing bodies of water very early in its history $(\sim 3$ billion years ago). However, any surface water was lost long ago.
It is probable that not all Earth-like planets in other solar systems have surface water. If they had characteristic geologic histories like Mars and Venus then they would not have standing bodies of water long enough to give rise to complex intelligent life forms. It is possible that plate tectonics and a magnetic field would be required which Mars and Venus do not have. We do not know what fraction of Earth-like planets in our galaxy currently has surface water. An optimistic figure might be about $80 \%$. A pessimistic figure is only $10 \%$. This low estimate assumes that most (90\%) Earth-like planets have had a geologic evolution similar to Venus or Mars. This estimate is probably too low. The unrealistic value is $5 \%$ that is almost surely too low.

\section{Earth-like planets where life originated [fl]}

Although water may be required for the origin of life, we do not know if life will always occur if it is present. The earliest most primitive life on Earth was prokaryotes (unicellular organisms whose cells lack a membrane-bound nucleus). They were present about 3.6 billion years ago $(\mathrm{Ga})$. However, the precursors to this primitive life have not been found so we cannot be sure how common it would be. If future missions to Mars determine that the organic compounds the Curiosity rover discovered are indeed due to life, then it would indicate that on a planet with surface water life should be common. If we assume that is the case then an optimistic figure for the presence of life on an Earthlike planet with surface water might be $100 \%$, and a pessimistic figure might be $50 \%$. An unrealistic value is $25 \%$

\section{Earth-like planets where complex life migrated to land [fld]}

This may sound very naïve, but if a planet was completely covered in water there would be no land for life to migrate, and probably no advanced technological life would evolve. Seventy percent of Earth is covered by water. On Earth there is intelligent life in the oceans in the form of dolphins and whales, but they do not have body parts (arms and hands) to develop technology. It is probable that for life to develop technology it must manipulate tools to acquire civilization and eventually technology. However, if plate tectonics are active on the planet there would probably be at least some land surfaces above sea level for life to migrate. An optimistic figure might be $95 \%$ and a pessimistic figure might by $50 \%$. The unrealistic value is only $25 \%$.

\section{Earth-like planets where intelligent life evolved [fi]}

If life emerged on a planet it is possible that in time it would evolve into more and more complex forms as it adapted to changing conditions. We have to define what intelligence is in order to estimate the probability that it will evolve. One comprehensive definition is: " $A$ very general mental capability that, among other things, involves the ability to reason, plan, solve problems, think abstractly, comprehend complex ideas, learn quickly and learn from experience." This definition certainly applies to humans, but it also applies to other species such as dolphins, chimpanzees, and whales, all of which are mammals.

It appears that on Earth intelligence is restricted to one Class of animals (mammals). On other planets this might not be the case. How long it takes for intelligence to evolve, or if it could occur in other Classes of life, is not known. It may have appeared in dinosaurs or other complex forms of life earlier in the geologic past. However, it is fairly likely that intelligence would appear given enough time and increasing complexity of the life. An optimistic estimate may be $80 \%$, and a pessimistic estimate may be $25 \%$. There is a large uncertainty in 
both numbers but it is likely to be somewhere between these values. An unrealistic value is $12 \%$.

\section{Earth-like planets where technological life developed [ft]}

Just because intelligent life evolves on a planet does not necessarily mean that intelligent life will automatically evolve a technology. It has taken 4.56 billion years for technological life to develop on Earth, and it still may not be developed on other planets that are younger or even older than Earth. It depends on whether the intelligent life develops appendages that can manipulate complex mechanisms. Although chimpanzees are intelligent, have hands and can manipulated objects in a crude way, they have not developed a technology. These uncertainties make it very difficult to estimate the percentage of planets with intelligent life that has technology. A very optimistic number may be $75 \%$, and a pessimistic value may be only $10 \%$. Certainly an unrealistic value is $5 \%$.

\section{Earth-like planets with current technological life [fc]}

This is by far the most difficult constraint to evaluate. It took 4.56 billion years for intelligent technological life to develop on Earth. Furthermore, that technological life would still not be here if it had not been for a mass extinction 66 million years ago that killed the dinosaurs and many other species. It is highly unlikely that technological life on other planets developed at the same time as ours. It may have developed much earlier or has not yet developed. The percentage of technological life estimated in the last constraint is for technological life that is present now. In this case it probably developed at or before our own technology. Since our technology only started about 250 years ago-a miniscule part of the geological time scale-the other technologies probably started well before ours. In this case they could be millions of years before our own, and, therefore, much more advanced. The other unknown is how long they survived in a technological state. For humans there are 6 ways that we may become extinct or at least end civilization and technology; four are natural occurring and two are suicidal:

- Rapid spread of a fatal, air-borne "Super" bacteria or virus resistant to all antibiotics (Natural).

- Impact of a large asteroid ( $10 \mathrm{~km}$ diameter) or comet (Natural).

- A gigantic eruption of lava like the Siberian or Deccan flood basalts or a catastrophic explosive eruption similar to the one that produced the Yellowstone region in the U.S. (Natural)
- Gamma-ray bust from a relatively nearby supernova. (Natural)

- Global warming to the catastrophic level $\left(\sim 6^{\circ} \mathrm{C}\right.$ above the preindustrial level). Currently we are causing the Earth to head in that direction (Suicidal).

- Full-scale atomic war (Suicidal).

All of these extinctions would happen in a geologically short period of time. Consequently, technological beings may only last a short time on a geologic time scale.

Remember that $99.9 \%$ of all species that lived on Earth are now extinct. Maybe our technology can prevent this from happening to us, but it could also kill us. On other planets with technological life there may be other ways to become extinct.

It may be rather rare that technological life is present today so an optimistic value may be only $10 \%$, and a very pessimistic value is just $1 \%$. Certainly an unrealistic value is $0.5 \%$. However, it is probable that most or all surviving technological life formed earlier than our own, and is probably much more advanced than our technology.

\section{Evaluation}

In Table 4 are the results of the estimated parameter values discussed in the previous section. It includes the "optimistic" and "pessimistic" values, plus a very "unlikely" estimate that is half the pessimistic percentages. Using the optimistic values gives an enormous number (300 million) of technological civilizations in our galaxy. Even the pessimistic values give a large number ( $\sim 41$ thousand). If the minimum size of the habitable zone is used then that number is about 20,500 .

The "unlikely" percentages are almost certainly too low but still give over 600 current technologies. If they were correct then 95\% of Earthlike planets in the habitable zone would not have surface water. This would mean that $95 \%$ of the planets would have had geologic histories like Mars or Venus with no plate tectonics. This is highly unlikely. It would also mean that $75 \%$ of those planets that did have surface water would be lifeless, and that $75 \%$ of the ones that did have life would probably be completely covered by water with no land above sea level. Again this is unlikely. Furthermore, 95\% of any intelligent life forms that evolved would not have developed a technology for the unlikely case.

\begin{tabular}{|l|l|l|l|l|l|l|}
\hline Parameter & $\begin{array}{l}\text { Optimistic } \\
\text { (percent) }\end{array}$ & Number & $\begin{array}{l}\text { Pessimistic } \\
\text { (percent) }\end{array}$ & Number & $\begin{array}{l}\text { Unlikely } \\
\text { (percent) }\end{array}$ & Number \\
\hline Surface Water & 80 & $5.28 \times 10^{9}$ & 10 & $6.6 \times 10^{8}$ & 5 & $3.3 \times 10^{8}$ \\
\hline Life & 100 & $5.28 \times 10^{9}$ & 50 & $3.3 \times 10^{8}$ & 25 & $8.25 \times 10^{7}$ \\
\hline Land Life & 95 & $5.02 \times 10^{9}$ & 50 & $1.65 \times 10^{8}$ & 25 & $2.06 \times 10^{7}$ \\
\hline Intelligent Life & 80 & $4.01 \times 10^{9}$ & 25 & $4.12 \times 10^{7}$ & 12 & $2.47 \times 10^{6}$ \\
\hline Technology & 75 & $3.01 \times 10^{9}$ & 10 & $4.12 \times 10^{6}$ & 5 & 123,750 \\
\hline Current Tech. Life & 10 & $3.0 \times 10^{8}$ & 1 & 41,250 & 0.5 & 619 \\
\hline
\end{tabular}

Table 4: This table lists the constraints, and the percentage and number of planets with the various parameters. The initial number of planets is 6.6 billion. 
Probably the most dire aspect of all the constraints in the "optimistic", "pessimistic" and "unlikely" cases is that between $90 \%$ and 99.5\% of all the technologic civilizations have become extinct. This would imply that we have a $90-99.5 \%$ probability of becoming extinct like the other $99.9 \%$ of species that once inhabited Earth. We are apparently now entering the 6th mass extinction so our species may now be on the path to extinction unless we take immediate action to prevent it [18]. If you feel more comfortable with a $50 \%$ value for the Current Tech. Life, then there would be 1.5 billion in the optimistic case, 2.06 million in the pessimistic case, and about 62,000 in the unlikely case. These values are almost surely too high.

For an initial value of either 3.3 or 6.6 billion Earth-like planets in the habitable zone of Sun-like stars, a value of only $2.5 \%$ for each parameter gives about 1 (1.6 and 0.8 ) technological society (us) in our galaxy. However, that percentage for all parameters is highly unlikely.

Furthermore, these estimates do not include planets in the habitable zones of red-dwarf stars that may number about 105 billion (Part 2). Although many of these would probably be bombarded with lethal radiation from their stars (Part 2), it is possible that some fraction would have a magnetic field strong enough to ward off the dangerous radiation. Also, the "pessimistic" estimates of $10 \%$ for surface water and $1 \%$ for current technological life are extremely low and are probably underestimated.

Therefore, it is highly probable we are not alone in our galaxy. There are probably hundreds to many thousands of technological societies presently in our galaxy. Also it is probable that almost all of these technologies are far more advanced than ours because their technologies probably started well before ours that began only yesterday on the geologic time scale (Tables 2 and 3).

For larger galaxies like Andromeda with about 1 trillion stars, the values would be over 3 times greater, e.g., the "pessimistic" value would be about 137,000 planets with technological beings, and over 2000 planets for the unrealistic case.

\section{Part 6: Contact with galactic technologic Life}

If there are presently somewhere between several million and 3,300 technologic civilizations in our galaxy, then most or all of them should be far more advanced than our own. Our technology only formed 250 years ago, which is 1.7 seconds ago on the geologic time scale relative to 1 year. They would have formed before our own technology and probably advanced to a stage that allowed them to communicate with other solar systems or even achieve inter-stellar travel. So where are they?

Assuming the planets with present technological life are uniformly distributed throughout the galaxy, then the average distance between them can be estimated by calculating the volume of the galaxy and using the following formulation: The galactic area $\left(\pi r^{2}\right)$ is about 9.5 billion $L Y^{2}$, and its volume $(\pi 2 t)$ is $9.5 \times 10^{12} L Y^{3}$, where the thickness $(\mathrm{t})$ is $1000 \mathrm{LY}$ and the radius $(\mathrm{r})$ is $55,000 \mathrm{LY}$. The cubic $\mathrm{LY}\left(\mathrm{G}_{\mathrm{v}}\right)$ per planet $(\mathrm{Hp})$ or $\mathrm{C}_{\mathrm{p}}$ is $\mathrm{G}_{\mathrm{v}} / \mathrm{Hp}$. The average diameter $(\mathrm{d})$ of the volume $\left(G_{v}\right)$ is: $d^{3}=C_{p} / 1.33 \pi$ or $d=3 \sqrt{ } \mathrm{Cp} / 1.33 \pi$. Then the average distance $(r)$ between planets with technological life is $1 / 2 \mathrm{~d}$. The number of planets $\left(\mathrm{H}_{\mathrm{p}}\right)$ is the number estimated in Table 4 . Table 5 below lists the number of planets with present technological life $\left(\mathrm{H}_{\mathrm{p}}\right)$ from Table 4 , the value of $C_{p}$, and the average distance $(r)$ between the planets in Light Years. However, the stars in our galaxy are not uniformly distributed. There are concentrations in the arms of the galaxy and other concentrations elsewhere. Since the Sun is in one of these arms there are more stars closer to our Sun than in the areas between arms. Therefore, the values listed in Table 5 are probably a maximum relative to distances from Earth.

The one completely unknown factor is the length of time technological life survives. In Part 5 the estimated survival was only $1 \%$ for the "pessimistic" case. Because Earth's time span of our technological civilization is so short on a geological time scale (Table 3 ), most of the present technological life in our galaxy must have occurred before ours. Their technologies could be thousands to millions of years older than ours. They may have developed sophisticated ways of detecting advanced life on other planets, and even means of interstellar travel.

\begin{tabular}{|l|l|l|}
\hline $\begin{array}{l}\text { Present Number of } \\
\text { Planets } \\
\begin{array}{l}\text { With Technological Life } \\
\left(\mathbf{H}_{\mathrm{p}}\right)\end{array}\end{array}$ & $\begin{array}{l}\text { Galaxy Volume } / \mathbf{H}_{\mathrm{p}} \\
\left(\mathrm{C}_{\mathrm{p}}\right)\end{array}$ & $\begin{array}{l}\text { Average Distance }(\mathbf{r}) \\
\text { between Technological } \\
\text { Life } \\
(\mathrm{LY})\end{array}$ \\
\hline $3.0 \times 10^{8}$ & 31,666 & 9.8 \\
\hline 41,250 & $2.3 \times 10^{8}$ & 190 \\
\hline $\mathbf{6 1 9}$ & $\mathbf{1 . 5 3 \times 1 0 ^ { 1 0 }}$ & $\mathbf{7 7 0}$ \\
\hline $310^{*}$ & $\mathbf{3 . 0 6 \times 1 0 ^ { 1 0 }}$ & $\mathbf{9 7 1}$ \\
\hline *Smaller habitable zone [4]. & & \\
\hline
\end{tabular}

Table 5: Average distance (r) between planets with technological life for a uniform distribution throughout the galaxy. The Bold values are highly unlikely.

How would a technological civilization detect Earth's technology? If they detected our radio transmission they would have to be within $\sim 100$ LY of Earth because our strong transmissions began 92 years ago (Figure 1f). However, if they could detect our night-lights then they may have detected our presence about 135 years ago when we began to light our cities at night using electricity (Figure 4a). If the civilization was very advanced they may have detected our gas street lamps about 200 years ago. For instance, if they were $\sim 190$ LY away as suggested by the pessimistic value of the estimates, then they would just now be receiving our faintest light output (Figure $4 \mathrm{~b}$ ). When or if they sent a radio/light signal to us it would not arrive at Earth until over $\sim 200$ years had passed. The unrealistic case (619 technological civilizations) would be on average $\sim 1,000 \mathrm{LY}$ away and will not receive our signal for 1000 years.

This illustrates the great difficulty of communicating with any civilization in our galaxy unless they were very close to us. Any advanced civilization beyond about 200 LY would not know we existed. However, if the optimistic estimate is correct then the average distance is only about $4 \mathrm{LY}$ and it is likely that we have already been detected. It is likely that they would be very much more advanced and possibly developed interstellar travel (Part 7 and Table 6). It is possible that they have not contacted us because the optimistic evaluation is too optimistic. If the pessimistic values are realistic then they may just be receiving our transmissions. 
Citation: Strom, Robert G (2015) We are not alone: Extraterrestrial Technological Life in our Galaxy. Astrobiol Outreach 3: 144. doi: $10.4172 / 2332-2519.1000144$

Page 16 of 18

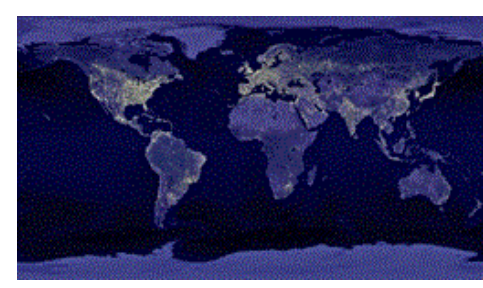

Figure 4(a): This composite image shows the Earth's lights at night. If a technological civilization could detect our electric lights that we began emitting about 135 years then they would have to be within $135 \mathrm{LY}$ of Earth. If they were advanced enough to detect gas lights that we started emitting about 200 years ago then they would have to be at or within $200 \mathrm{LY}$ of Earth (Figure 4b).

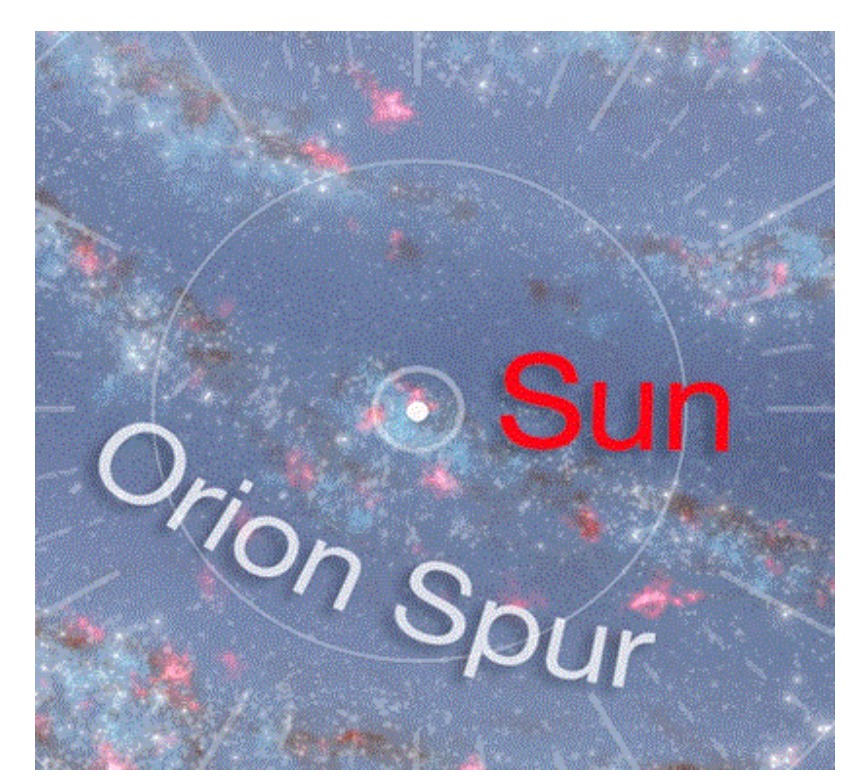

Figure 4(b): The location of this part of the galaxy is shown in Figure 1e. The white dot in the center has a radius of $200 \mathrm{LY}$ that is the distance our early street gaslight has traveled. An advanced civilization would have to be within that dot to detect the light at the present time.

\section{Part 7: Technological aliens}

It took 4.56 billion years for human technology to evolve on Earth. Earth's human technology began 250 years ago that is equivalent to 1.7 seconds ago on the geologic time scale normalized to one year (Table 3). It is highly unlikely that any present alien technology evolved on the same time scale. All of them are probably thousands to millions, or even tens of millions of years, older than our technology. If alien technologies advanced at about the same rate as ours they would be far more advanced. If our technology kept advancing at its current rate, imagine what it would be 1,000 years from now.

It is also likely that they do not look anything like humans. They probably have appendages to manipulate mechanisms, and eyes, noses, mouths and ears that allow them to see, hear, breath, and obtain nourishment. However, their appearance is probably very different from our own. Apes and monkeys have all these features but look very different than humans. They could also have four arms and four legs. Maybe they could even be as different as the statue shown in Figure 5.

Aliens from other planets may have very different life expectancies. Our evolution has taken place on a planet that rotates with a period of 1 day. We experience day and night about every $12 \mathrm{hrs}$. We rest at night and are active during the day. This may contribute to our life expectancy although other factors surely contribute. If aliens evolved on a planet that has a rotation and orbital period that produces very long days and nights then it may result in a life expectancy much longer than ours. Also it is possible that they could produce body parts and replace body organs and tissue so that they could live thousands of years or even forever.

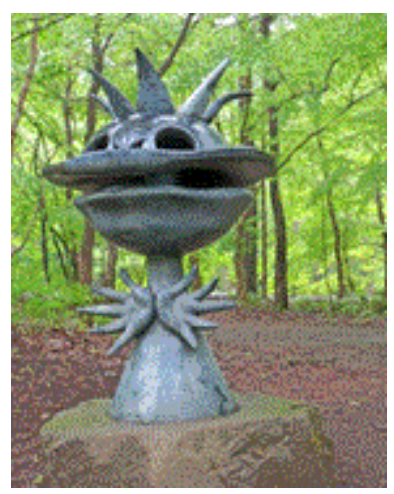

Figure 5: A statue on the grounds of the Keiryukan Hotel, in Oirase, Japan.

It is very possible that several of these very advanced technologies have developed spacecraft capable of achieving interstellar travel at near light speed, or maybe ever greater. They may have learned how to use "Dark Energy" and/or "Dark Matter" to attain speeds greater than the speed of light. Today we do not understand these entities although Dark Matter comprises over 95\% of the Universe. Table 6 is an attempt to estimate the number of civilizations that have attained interstellar travel at the present time. Even the "pessimistic" estimate of $1 \%$ suggests a minimum of over 400 , and that is probably too low.

\begin{tabular}{|c|c|c|c|c|}
\hline \multirow{2}{*}{\multicolumn{2}{|c|}{$\begin{array}{l}\text { Present Technological Life in } \\
\text { the } \\
\text { Habitable Zones of Sun--like } \\
\text { Stars }\end{array}$}} & \multicolumn{3}{|c|}{$\begin{array}{l}\text { Number of Civilizations with Present } \\
\text { Interstellar Travel }\end{array}$} \\
\hline & & $\begin{array}{l}50 \% \\
\text { Civilizations }\end{array}$ & $\begin{array}{l}25 \% \\
\text { Civilizations }\end{array}$ & $\begin{array}{l}1 \% \text { of } \\
\text { Civilization }\end{array}$ \\
\hline "Optimistic" & $3.0 \times 10^{8}$ & $1.5 \times 10^{8}$ & $7.5 \times 10^{7}$ & $3.0 \times 10^{6}$ \\
\hline "Pessimistic" & 41,250 & 20,624 & 10,312 & 412 \\
\hline "Unlikely" & 619 & 309 & 155 & 6 \\
\hline
\end{tabular}

Table 6: Estimates of the number of civilizations with present interstellar travel for 50,25, and 1 percent possibilities. These estimates are based on the number of technological civilizations estimated in Table 4.

As the space travelers approached the speed of light their time would slow so they would not age as much as on their home planet. They could visit solar systems many light years away. For instance, they 
could travel to a solar system $500 \mathrm{LY}$ away and only age maybe about 5 or 10 years depending on how close they came to the speed of light. If they travelled to a solar system $500 \mathrm{LY}$ away and returned to their home planet, they would have only aged about 10 or 20 years. However, because the roundtrip time between the planets is 1,000 LY, their planet would have aged about 1,000 years. If they sent a message to their home planet from the one they were visiting it would arrive only a few years before they did. This is one of the tight constraints about communication and travel in our galaxy. However, if they were very far advanced they may have discovered a way of travelling faster than the speed of light. Even if we could travel at the speed of light and time would slow to a crawl for the crew, by the time we returned to our home planet it would have aged hundreds to thousands of years depending on the distance of the planet we were visiting. Therefore, the civilization we left behind would have changed greatly or become extinct.

It is also possible that they have spaceships that are self-sufficient and no longer require supplies from, or communication with, their home planet. If civilization lasted to the end of their sun's life, then they would have to abandon their planet and find somewhere else to live; that could be another planet or space itself.

This all may sound like science--fiction, but we just cannot imagine what kind of technology very advanced technologies would have developed over a period of thousands of years or more. Imagine you are living at the beginning of the Industrial Revolution when the main form of transportation was by horse. Suppose someone predicted that in 250 years $(2015)$ we would have machines that would drive us along roadways spanning our continent. We could drive the equivalent distance from Tucson to Phoenix in about an hour. Two hundred and fifty years ago it would have taken over a day on horseback or stagecoach to travel that distance. The person also predicted that we would have machines that would fly like the birds but at very great speeds, and that we could travel from New York to London in one of these machines in just a few hours. The person would not predict electronic technology that includes computers, radio, TV, and the Internet because he would not have the foggiest idea what they were. The people in those days would think he was insane and probably send him to an asylum or worse.

Is it possible that we have been visited by one of the technological societies in our galaxy? This is a highly uncertain and controversial possibility. However, there are a number of UFO (Unidentified Flying Objects) sighting that have never been adequately explained.

One sighting occurred on Oct. 27, 1954 at a soccer game in Florence, Italy when 10,000 people viewed two oval objects in the sky. Even the players stopped the game to look at the objects. Also that day in Tuscany, and several days thereafter, similar objects were observed.

Another sighting occurred in November 1986 when a cargo flight of Japan Airlines (flight 1628) observed a bright object "larger than an aircraft carrier" near them on their approach to Anchorage, Alaska. The Anchorage airport reported to the JAL flight that there were no commercial airline flights in the area. They requested the flight to make a circle to see if the object would follow them. They made the circle and the object did follow them. The object was also observed by both airport and military radar at the same locations and motions described by the aircraft crew.

There have been many other unexplained sightings, but these two are particularly hard to explain. If we have been visited by one or more alien technologies then there are several possibilities concerning their civilization. If they are here because they detected our light or radiowave radiation, then their home planet must be within a maximum of $200 \mathrm{LY}$, and probably more likely $100 \mathrm{LY}$ from Earth. This would suggest that technological civilizations are more frequent than those estimated from the 600 and 41,000 planets. If the UFO sighting in Italy 60 years ago is correct then their home planet is within about $40 \mathrm{LY}$ suggesting technological life is very common and the optimistic value of about 300 million is more likely. However, technological life within a few light years may be a possibility.

It is possible that the aliens are just exploring planets that are similar to theirs and they happened to discover ours, just as the marine explorations in 14th and 15th centuries were discovering new lands. In this case they could come from anywhere in our galaxy. They also may have evolved to the point where they no longer need a home planet and just exist in a fleet of extremely advanced spacecraft. If this is the case then maybe they are originally from one of the Sun-like stars that were formed near the time of origin of our galaxy (13.2 billion years) and are now extinct.

The main question is why they have not contacted us by either communication or direct contact. Probably the first thing they would do before direct contact is to observe us for some time to learn our characteristics and behavior. We would probably do the same thing. If they were peaceful beings, they would probably be horrified by the behavior of humans; violence (including World Wars), destruction of our environment, pollution of our water and atmosphere, and the massive emission of greenhouse gases that are changing our planet. They would be able to view our television programs, translate our languages, and directly observe our many wars that killed millions including the Holocaust where one group of people systematically exterminated millions of others. They would learn that our history is one of war. They would witness the ongoing fighting in Iraq, Syria, Libya and Afghanistan, and the hideous atrocities that have occurred in those countries. They would also see the personal crimes and violence among ourselves that occur daily. Their only conclusion must be that we are a very violent and dangerous species. The one thing that may favorably impress them is our artistic ability.

Of course, discussion of aliens visiting the Earth is complete speculation, but it should at least be discussed when dealing with technological life in the galaxy. If it is not happening now, it could happen in the future.

\section{Conclusions}

The evaluation of constraints on the number of technological civilizations in our Milky Way galaxy for planets in the habitable zone of stars like our Sun indicates that under the most pessimistic considerations we are not alone. This evaluation only considered the estimated 6.6 billion Earth-sized planets in the habitable zones of Sunlike stars. It did not considered the estimated 105 billion Earth-like planets in the habitable zones of Red Dwarfs because of the probability they were strongly subjected to intense radiation from their stars. The estimates indicate that a bare minimum of about 600 technological civilizations currently exists in our galaxy. However, it is more likely there are well over 40,000 technologies. The evaluations of present technological civilizations indicate that we have over a $90 \%$ probability of becoming extinct. The fact that we are already entering the 6th mass extinction caused by humans suggests we already may be headed that way. 
Citation: Strom, Robert G (2015) We are not alone: Extraterrestrial Technological Life in our Galaxy. Astrobiol Outreach 3: 144. doi: $10.4172 / 2332-2519.1000144$

Page 18 of 18

It is highly unlikely that any present alien technology evolved on the same time scale as ours (Table 3). They almost surely started thousands to millions of years before ours and are, therefore, far more advanced.

Contacting these civilizations is extremely difficult because of the great distances within our galaxy. Our current SETI project is searching for a signal from any advanced civilization. It is a very worthwhile endeavor and should be continued. However, once we hear from that civilization it will be difficult to contact them unless they are very close to Earth. There is also a discussion among space scientists about directly sending a message to stars with detected planets. This also is very controversial because of the possibility they would visit us in the future with possibly adverse consequences.

If UFOs are real (highly speculative) then we have already been observed by at least one of these extremely advanced civilizations. We should make a concerted effort to determine if they exist or not.

\section{References}

1. Petigura EA, Howard AW, Marcy GW (2013) Prevalence of Earth-sized planets orbiting Sun- like stars. Proc Natl Acad Sci USA. 110: 19273-19278.

2. Robitaille TP, Whitney BA (2010) The present-day star formation rate of the Milky-Way determined from Spitzer-detected young stellar objects. Astro J Letters 710: L11-L15.

3. Campante T L, Barclay T, Swift J J, Huber D, Adibekyan V Zh, et al. (2015) An ancient extrasolar system with five sub-Earth-size planets. Astrophysics 799.

4. Kasting JF, Harman CE (2013) Extrasolar planets: Inner edge of the habitable zone. Nature 504: 268-271.

5. Fressin F, Torres G, Charbonneau D, Bryson S T, Christiansen J, et al. (2013) The false positive rate of Kepler and the occurrence of planets. Astrophys J 766: 81-100.

6. Johnson J (2014) Warm planets orbiting cool stars. Physics Today 67: 31-36.
7. Quintana E, Barclay T, Raymond S N, Rowe J F, Bolmont E, et al. (2014) An Earth-sized planet in the habitable zone of a cool star. Science 344: 277-280.

8. Dressing C, Charbonneau D (2013) The occurrence rate of small planets around small stars. Astrophys J 767: 95.

9. Leconte J, H Wu, Menou K, Murray N (2015) Asynchronous rotation of Earth-mass planets in the habitable zone of lower-mass stars. Science 347: 632-635.

10. Cohen O, Drake JJ, Glocer A, Garraffo C, Poppenhaeger K, et al. (2014) Magnetospheric structure and atmospheric joule heating of habitable planets orbiting M-Dwarf stars. Astro Phys.

11. Strom RG, Malhotra R, Xiao Z, Ito T, Yoshida F, et al. (2015) The inner solar system cratering record and the evolution of impactor populations Res Astro and Astrophys 15: 407-434.

12. Strom RG, Schaber GG, Dawson DD (1994) The Global Resurfacing of Venus. Jour Geophys Res 99: 10899-10926.

13. Arkani-Hamid A, Schaber G G, Strom R G (1993) Constraints on the thermal evolution of Venus inferred from Magellan data. J Geophys Res 98: 5309-5315.

14. Trail D, Mojzsis SJ, Harrison TM (2007) Thermal events documented in Haden zircons by microprobe depth profiles. Geochem Cosmochem Acta 71: 4044 .

15. Cleeves LI, Bergin EA, Alexander C, Fujun Du, Graninger D, et al. (2014) The ancient heritage of water ice in the solar system. Science 345: 1590-1593.

16. Richards M, Alvarez W, Self S, Karlstrom L, Renne P R, et al. (2015) Triggering the largest Deccan eruptions by the Chicxulub impact. Geol Soc Amer Bulletin.

17. Schoene B, Samperton K M, Eddy M P, Keller G, Adatte T, et al. (2015) U$\mathrm{Pb}$ geochronology of the Deccan Traps and relation to the endCretaceous mass extinction. Science 347: 182-184.

18. Ceballos G, Ehrlich P, Barnosky A, Garcia A, Pringle R, et al. (2015) Accelerated modern human--induced species losses: Entering the sixth mass extinction. Sci Adv 1: 5. 